bioRxiv preprint doi: https://doi.org/10.1101/2021.06.09.447763; this version posted August 12, 2021. The copyright holder for this preprint (which was not certified by peer review) is the author/funder, who has granted bioRxiv a license to display the preprint in perpetuity. It is made available under aCC-BY-ND 4.0 International license.

\title{
Clock time: a foreign measure to brain dynamics
}

\author{
Authors \\ Sander van Bree ${ }^{1,2 *}$, María Melcón ${ }^{3}$, Luca Kolibius ${ }^{1,2}$, Casper Kerrén ${ }^{4}$, Maria Wimber ${ }^{1,2}$ \& Simon \\ Hanslmayr ${ }^{1,2}$ \\ *Correspondence: sandervanbree@gmail.com
}

\begin{abstract}
Affiliations
${ }^{1}$ Centre for Cognitive Neuroimaging, Institute for Neuroscience and Psychology, University of Glasgow, Glasgow, United Kingdom

${ }^{2}$ Centre for Human Brain Health, School for Psychology, Birmingham, United Kingdom

${ }^{3}$ Department of Biological and Health Psychology, Autónoma University of Madrid, Spain

${ }^{4}$ Center for Adaptive Rationality, Max Planck Institute for Human Development, Berlin, Germany
\end{abstract}

\begin{abstract}
Human thought is highly flexible and dynamic, achieved by evolving patterns of brain activity across groups of cells. Neuroscience aims to understand cognition in the brain by analysing these intricate patterns. Here, we argue that this goal is impeded by the time format of our data - clock time. The brain is a system with its own dynamics and regime of time, with no intrinsic concern for the human-invented second. A more appropriate time format is cycles of brain oscillations, which coordinate neural firing and are widely implicated in cognition. These brain dynamics do not obey clock time - they start out of tune with clock time and drift apart even further as oscillations unpredictably slow down, speed up, and undergo abrupt changes. Since oscillations clock cognition, their dynamics should critically inform our analysis. We describe brain time warping as a new method to transform data in accordance with brain dynamics, which sets the time axis to cycles of clocking oscillations (a native unit) rather than milliseconds (a foreign unit). We also introduce the Brain Time Toolbox, a software library that implements brain time warping for electrophysiology data and tests whether it reveals information patterns in line with how the brain uses them.
\end{abstract}

\section{Glossary}

\begin{tabular}{|c|c|}
\hline Brain oscillations & Rhythmic fluctuations of brain activity generated by populations of cells \\
\hline Brain time & Time as sequences of cycles of a coordinating brain oscillation \\
\hline Brain time warping & Algorithm that employs dynamic time warping to transform electrophysiology data in accordance with brain time dynamics \\
\hline Brain time toolbox & Software library that implements brain time warping and tests its effects \\
\hline Clock time & Time as sequences of seconds \\
\hline Dynamic time warping $(D T W)$ & Algorithm that can measure the similarity between signals and minimize their difference \\
\hline Frequency & Number of cycles per time window (typically a second) \\
\hline $\begin{array}{l}\text { Linear Discriminant Analysis } \\
(L D A)\end{array}$ & $\begin{array}{l}\text { Machine learning method that maximizes the separability between two classes of data by applying linear transformations to } \\
\text { it }\end{array}$ \\
\hline Local Field Potential $(L F P)$ & The electric potential recorded from extracellular space around cells \\
\hline Neural signature & Brain activity that systematically correlates with, in this context, a cognitive process \\
\hline Non-stationarity & $\begin{array}{l}\text { A signal is non-stationary when it undergoes spectral changes over time. We focus on frequency drift, variable starting } \\
\text { phases, and phase jumps. }\end{array}$ \\
\hline Periodicity & Fluctuating patterns of a neural signature \\
\hline Phase & $\begin{array}{l}\text { Metric to indicate the specific point in the cycle of an oscillation. Two oscillations are in phase when (for example) their } \\
\text { peaks align. }\end{array}$ \\
\hline $\begin{array}{l}\text { Temporal Generalization } \\
\text { Matrix }(T G M)\end{array}$ & $\begin{array}{l}\text { Representation of how a classifier trained to separate classes of data on one timepoint performs on other timepoints. When } \\
\text { a classifier generalizes, it indicates the neural signature remains stable. }\end{array}$ \\
\hline Warping path & Representation of how two signals need to be resampled to minimize their difference \\
\hline Warping source & Data structure containing potential coordinating brain oscillations used for brain time warping \\
\hline
\end{tabular}



made available under aCC-BY-ND 4.0 International license.

\section{Studying dynamic cognition}

Everyday tasks involve a plethora of cognitive functions that operate dynamically in tandem. Something as mundane as taking notes during a meeting or battling your friend in a video game requires attention, motor activity, perception, memory, and decision-making, each evolving over time. How does the brain achieve dynamic cognition? To answer this question, neuroscientists closely study how brain activity unfolds from one moment to the next using temporally precise neuroimaging methods. These include electroencephalography (EEG), magnetoencephalography (MEG), and single and multi-unit recordings - grouped together under the term electrophysiology.

\section{Seconds are foreign to the brain}

In a typical electrophysiology study, neuroscientists first probe cognition by introducing an experimental manipulation. For example, an attention researcher might introduce a set of moving dots. Then, to understand cognition in the brain, they perform a series of analyses on the recorded data. They might study changes in scalp topography over a second of data, apply machine learning to characterize how the representation of the dots evolves, or perform any other time-dependent analysis.

Critically, from the raw output of neuroimaging devices to the analysis of recorded brain signals, time is operationalized as clock time - sequences of milliseconds. We claim that clock time, with all its benefits for human affairs, is generally inappropriate for neuroscience. This is because clock time is defined by us and for us, based on how long it takes for Earth to rotate its axis. The brain itself, however, employs its own regime of time, dictated by its own dynamics. As such, the brain is indifferent to how many milliseconds, seconds, minutes, or hours have passed unless it is expressly relevant for specific behaviour, such as maintaining circadian rhythms [1] or tracking a time-dependent reward [2]. Instead, the brain is concerned with coordinating communication between cells in a delicate time-sensitive manner, such as sending information at one moment and receiving feedback signals at the next. Hence, the brain's intrinsic time format - brain time - is dictated by the internal processes that clock brain activity.

\section{Cycles as the brain's native unit}

What mechanism clocks brain activity? A defining feature of the brain is that its activity waxes and wanes [3], pointing to a central role of brain oscillations. Brain oscillations are wellgeared to structure brain activity. For one, each cycle of an oscillation contains a window of excitability where cells are more likely to fire $[4,5]$. Moreover, oscillations vary in their frequency, meaning the excitability windows vary in duration. The functional role of oscillations has been shown in a wide array of cognitive functions, including attention $[6,7,8]$, perception (auditory [9,10], visual $[11,12]$, tactile $[13,14]$ ), action $[15,16]$, memory $[17,18,19]$, and decision-making $[20,21]$. Together, this situates brain oscillations as the brain's clocking mechanism, clustering brain activity in flexible ways to organize dynamic cognition. The brain's base unit of time then, are the cycles of oscillations that coordinate neural firing, not the milliseconds with which we format our data.

The problem: clock and brain time are usually out of tune Why does it matter that we use a foreign time format? When neuroscientists study dynamic cognition, they repeat measurements across trials, resetting their stopwatch at the start of each. However, some oscillations do not reset [22,23]. Even when most do, oscillations evolve continuously in frequency and show jumps in phase (Figure 1). Thus, besides a potential mismatch between clock and brain time from the get-go caused by variable starting phases, the disharmony between the dimensions accumulates due to frequency drift and phase jumps. These are prime examples of eccentricities in brain dynamics, formally called non-stationarities (and there are more [24]). Their presence makes clock time an ill-suited format to study temporal patterns of dynamic cognitive function - it distorts how the brain itself carries information forward in time.

To demonstrate this point, take again the case of spatial attention. Studies show that alpha oscillations (8 to 12 Hertz; $\mathrm{Hz}$ ) in parietal regions orchestrate the dynamics of spatial attention $[25,26]$. If these oscillations vary in their starting phase across trials, then the neural patterns of spatial attention will vary along with it. Likewise, if the oscillations slow down in frequency, the patterns slow down too. If a researcher is interested in, say, decoding the locus of covert attention in the visual field over time, it would muddy the waters to do so in clock time, ticking away with its equal periods. The slowing down of alpha oscillations means brain time falls behind relative to clock time, so analysing the data in its default format yields a sped-up readout of attention's true pattern. Instead, we argue the dynamics of clocking oscillations should heavily inform data analysis. As a general mantra, the optimal approach to analyse the brain, like any other system, is with recourse to its own dynamics - from inside out $[27,28]$ (Box 1).

The problem of disharmony does not end here. Neuroscientists repeat measurements across participants to establish whether effects found in the data are representative
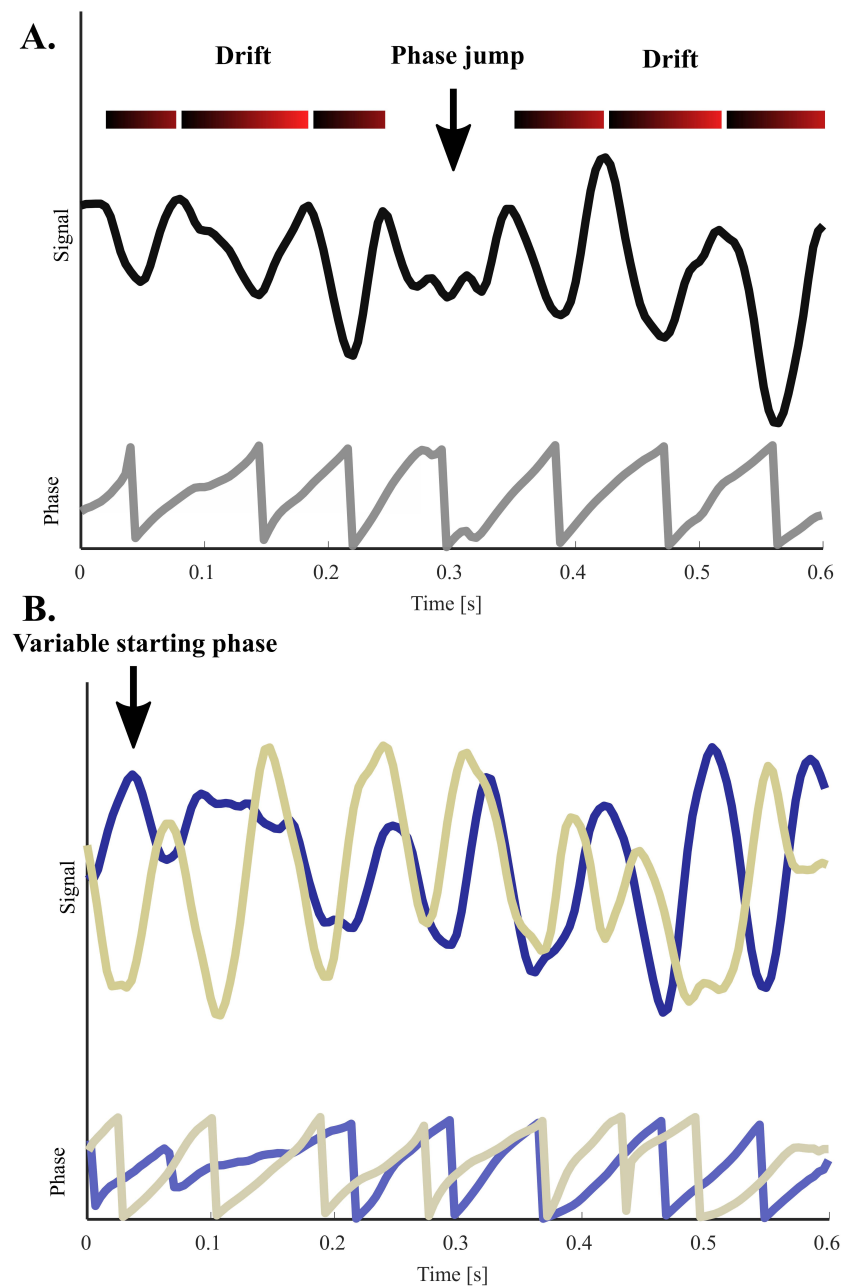

Figure 1: Sources of disharmony between clock and brain time Brain oscillations are nonstationary, which causes a non-correspondence or "disharmony" between the brain's internal dynamics and clock time. (A) An oscillation shows frequency drift and a spontaneous jump in phase, resetting itself. (B) Two oscillations with different starting phases. The blue oscillation starts with a rising phase, while the sand-coloured oscillation starts with a falling phase. The top rows of each panel show the amplitude fluctuations of oscillations, while the bottom rows show the phase. 


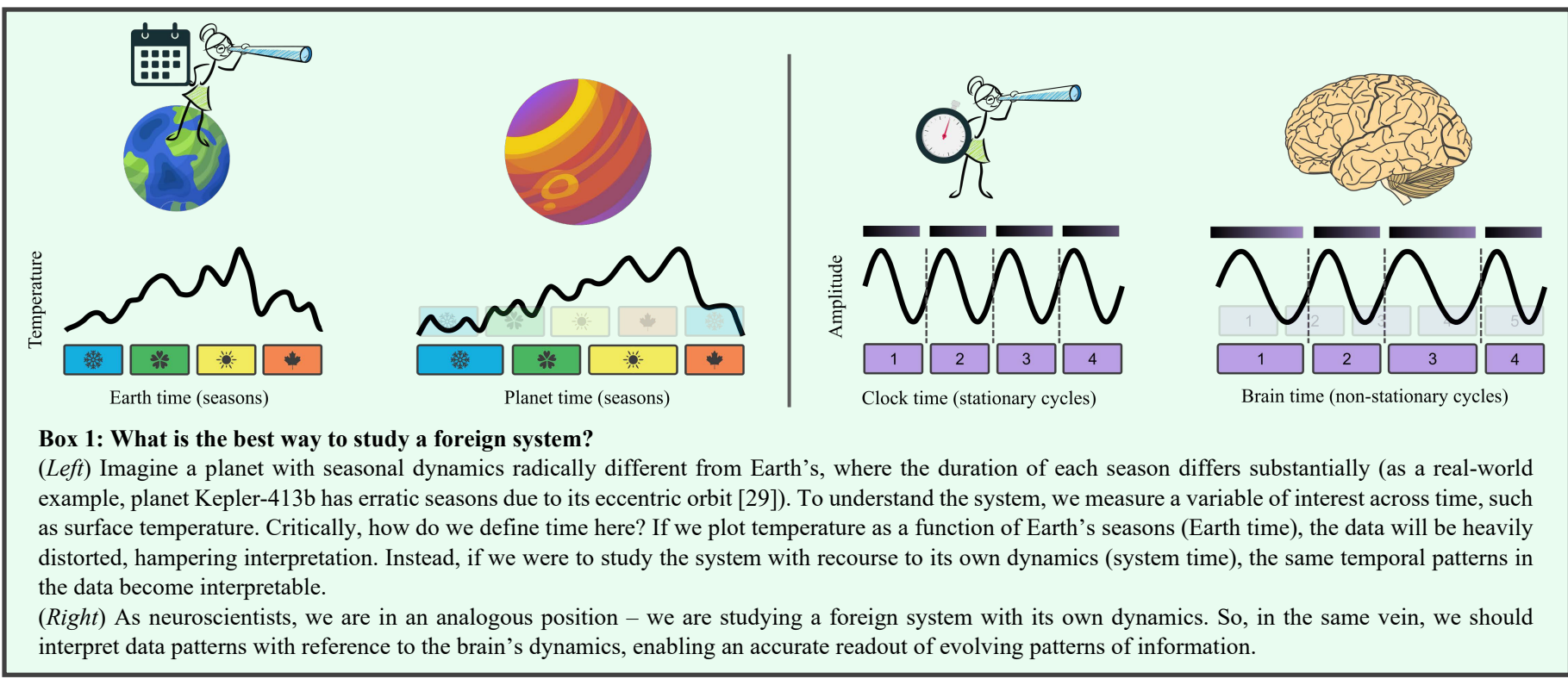

and statistically robust. But different brains have different dynamics, resulting in disharmony across brains too. In the attention experiment, it is highly relevant that the clocking alpha oscillations differ in frequency from person to person [30] (Figure 4D), as it means the patterns differ too. Looking for evolving patterns of spatial attention by averaging across participants is like asking when spring turns summer in a solar system that contains diverse planets - it only makes sense after correcting for individual dynamics.

\section{A solution: retuning clock and brain time by warping}

Disharmony between clock and brain time impedes analysis within and across brains. How can we account for it? We introduce brain time warping as a method to (1) identify segments where clock and brain time fall out of tune, and to (2) adapt the data in such a way to reduce their difference metaphorically winding back clock to brain time (Figure 2). Brain time warping incorporates an algorithm called dynamic time warping (DTW), which characterizes the similarity of two signals $[31,32]$. DTW computes a warping path, which shows how the samples of each signal need to be transformed to optimize their alignment. For brain time warping, those signals are clock and brain time (Figure 2A).

How are clock and brain time operationalized? Brain time can be characterized as the phase of the oscillations hypothesized to orchestrate a process' dynamics, with its variable starts, drift, and phase jumps. Clock time can be characterized as the phase of a stationary sine wave, fluctuating away faithfully to seconds. Such a signal is what brain time would look like without the three sources of disharmony; here milliseconds and cycles map directly onto each other. (For example, in Figure 2A, multiples of 100 milliseconds correspond to each zero-crossing of the clock time signal.)

DTW highlights during which samples clock and brain time fall into disharmony, and this warping path can be used to transform the original data. Concretely, at samples where the warping path suggests brain time needs to repeat itself before ramping back up to clock time, brain time warping repeats samples in the original data (Figure 2C). Looping back to our attention study, at segments where DTW indicates alpha oscillations slow down, brain time warping stretches the data in an attempt to bring its structure closer to the true dynamics of spatial attention.

Brain time warping loops over trials, continuously correcting disharmony by applying the warping path cycle by cycle. In effect, brain time warping adapts data to the dynamics of an oscillation of interest in a data-driven way. The result is a dataset in brain time rather than clock time, and as such, the time axis has changed from seconds to cycles. As the data is

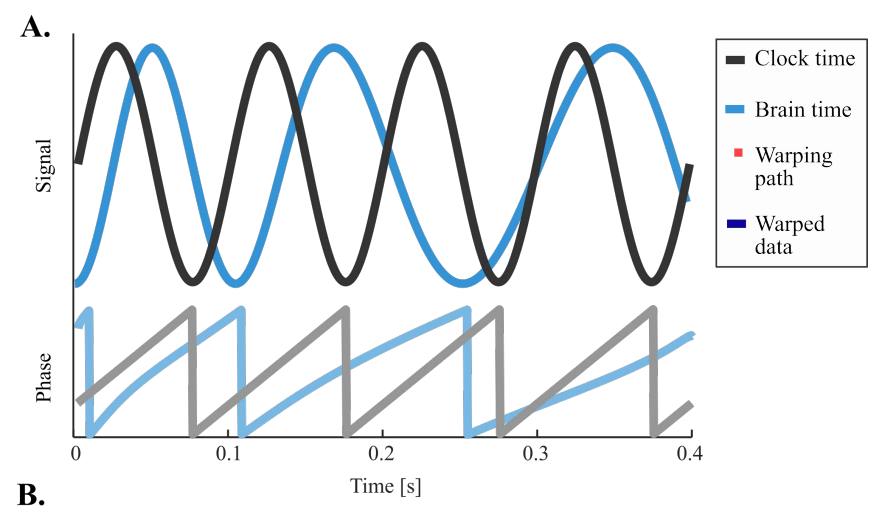

B.

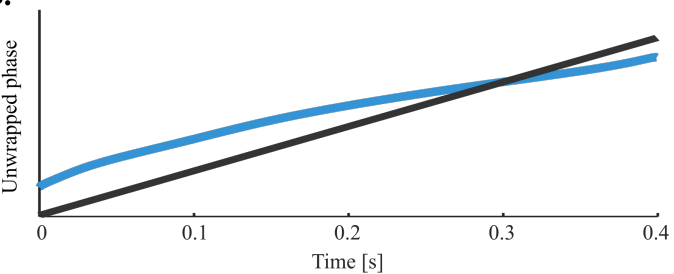

C.

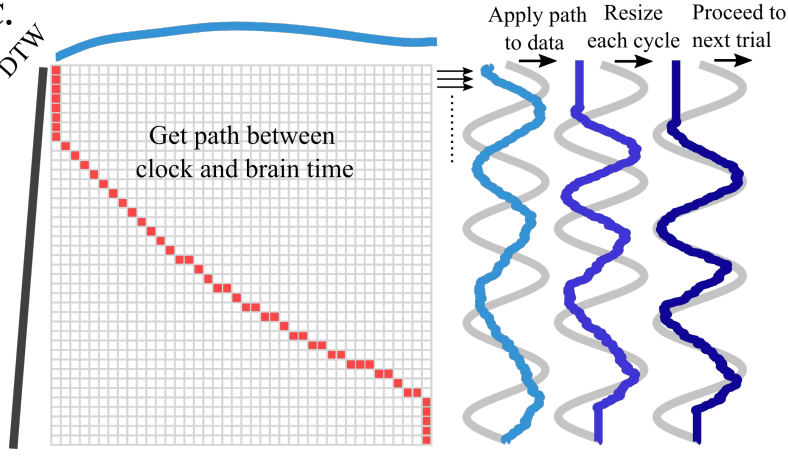

Figure 2: Brain time warping between clock and brain time

(A) Brain time starts in its rising phase and slows down its frequency over the course of the trial, both causing a mismatch to clock time (defined as a stationary signal fluctuating in sync with a researcher's stopwatch). (B) To facilitate warping, the phase of clock and brain time are unwrapped, meaning phase is computed without cycle resets. (C) DTW calculates a warping path that minimizes the difference between the dimensions. Cycle by cycle, the path is applied to the input electrophysiology data, transforming its dynamics in accordance with the brain's dynamics. To enable alignment of brain time across trials, the data of each cycle is resized to a constant number of samples. The previous steps are repeated for all remaining trials. Upon completion, the data's time axis is changed from seconds to cycles of brain time. The data is no longer in clock time, but in brain time. 
bioRxiv preprint doi: https://doi.org/10.1101/2021.06.09.447763; this version posted August 12, 2021. The copyright holder for this preprint (which was not certified by peer review) is the author/funder, who has granted bioRxiv a license to display the preprint in perpetuity. It is made available under aCC-BY-ND 4.0 International license.

referenced to individual dynamics, it also becomes easier to look for temporal patterns across brains. In the Supplementary Material, we introduce a MATLAB (The MathWorks, Natick, Massachusetts, USA) toolbox that implements brain time warping and tests its effects - the Brain Time Toolbox. This software library was built for electrophysiology data structures, including EEG, MEG, and single and multi-unit recordings. From here on out, each result we display was obtained using the toolbox.

\section{Brain time warping unveils dynamic neural patterns}

We demonstrate the effects of brain time warping in three datasets - one simulated, one from rodent single- and multi-unit recordings, and one from human EEG recordings. The premise of each analysis is the same. We separate two classes of data which we predict differ in brain activity only with respect to the cognitive process under investigation. This difference provides a classifier with a neural signature of cognition which it can use to predict what class held out data belong to. How the classifier performs over time thus informs us of dynamic

\section{Left (class 1)}

A.

C.

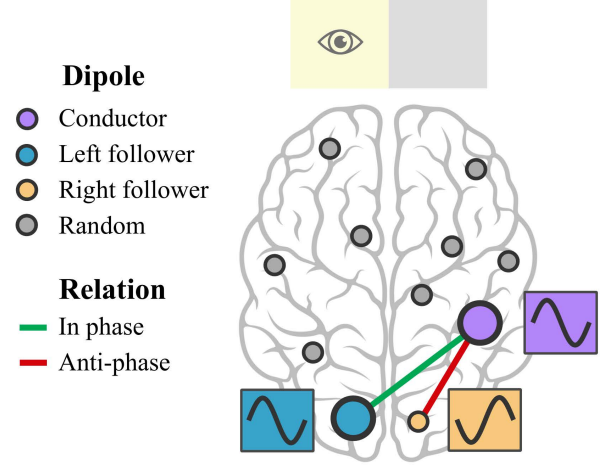

Example participant
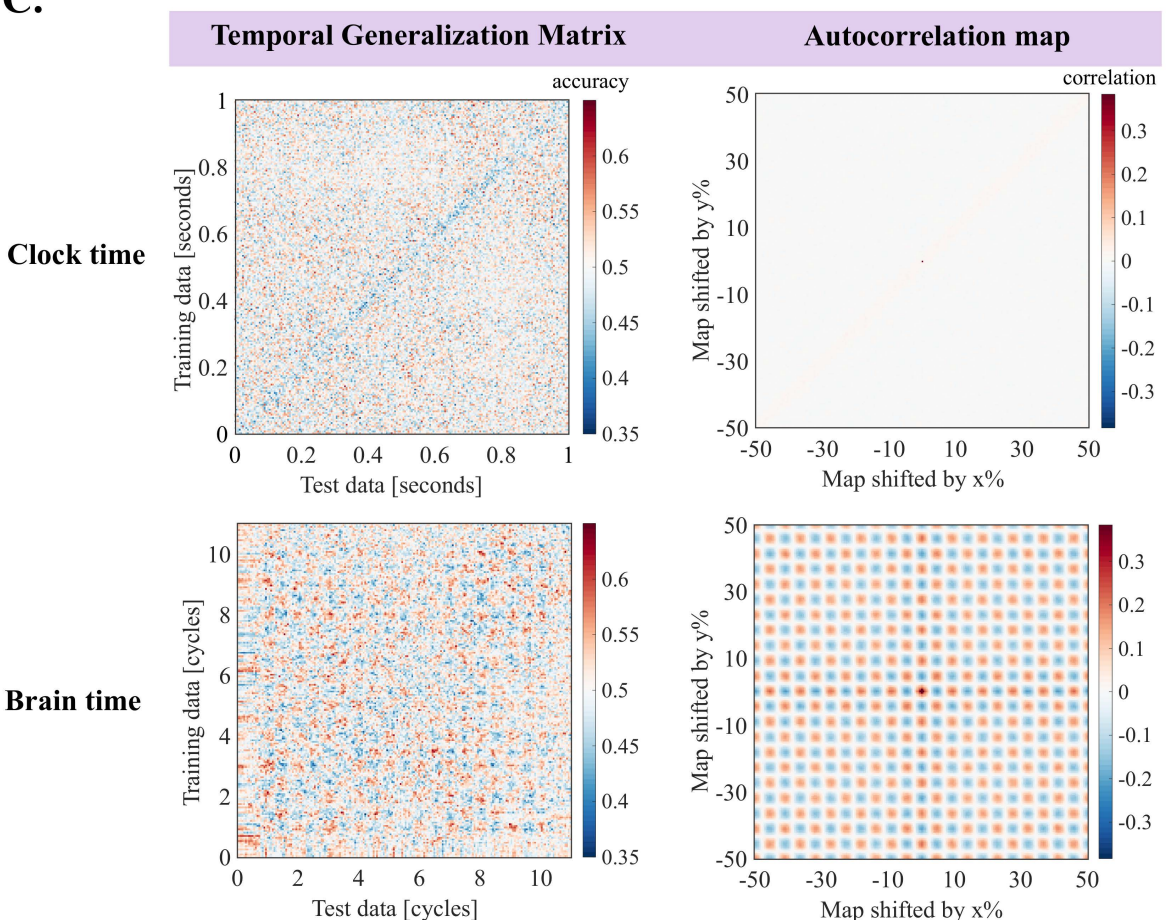

B.
(2)

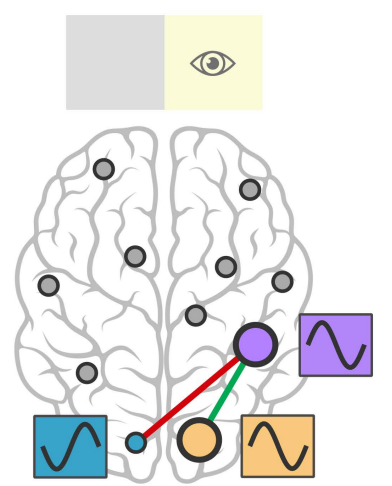

Right (class 2)
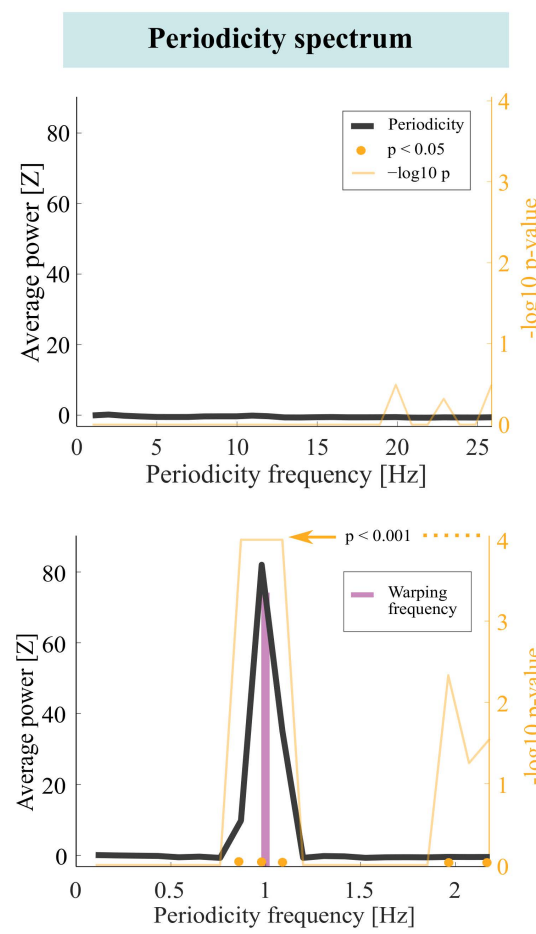

Figure 3: Brain time warping unveils ground truth patterns in simulated data

(A) To establish whether brain time warping facilitates the readout of dynamic brain patterns, we simulated a basic attentional spotlight model with dipoles oscillating at alpha (8 to $12 \mathrm{Hertz}[\mathrm{Hz}]$ ). One parietal conductor dipole "conducted" two follower dipoles, one in each visual cortex. We applied a linear discriminant analysis to differentiate two classes of data: covert attention to the left and right hemifield. These conditions differed in brain activity because of contralateral alpha inhibition and the phase relation between dipoles, constituting attention's neural signature. To introduce disharmony between clock and brain time, we added frequency drift and variable starting phases to the dipoles. (B) We created $\mathrm{n}=10$ datasets with a random individual alpha frequency, each serving as one virtual participant. Next, we tested for periodic patterns in the classifier's temporal generalization matrix (TGM), which represents how the classifier's performance generalizes across time. (C) In clock time, an example participant's TGM shows no periodic structure, nor does the TGM's autocorrelation map (top left and middle). In contrast, the TGM of brain time data shows periodic structure which becomes qualitatively striking in its autocorrelation map (bottom left and middle). We quantified periodicity by applying a fast Fourier transform over all rows and columns of TGMs. Then, we perform second-level statistics by comparing empirical periodicity with permuted periodicity (obtained by shuffling class labels). Only brain time spectra show significant periodicity at the warping frequency ( $p<0.001$ ) and its first harmonic $(\mathrm{p}<0.05)$, demonstrating brain time warping corrects for disharmony and unveils the ground truth pattern (bottom and top right). (General) Brain time periodicity spectra are referenced to the brain's own dynamics. Specifically, $1 \mathrm{~Hz}$ represents periodicity at the warping frequency of each participant (and $0.5 \mathrm{~Hz}$ at half of it), normalizing spectra to participants' individual alpha. We corrected for multiple comparisons (using false discovery rate; FDR [33]), except at specific frequencies in the brain time spectra at which we hypothesized classifier periodicity $(0.5 \mathrm{~Hz}, 1 \mathrm{~Hz}, 2 \mathrm{~Hz} ; 1 \mathrm{~Hz}$ remains significant when applying FDR $)$. All analyses were performed using the Brain Time Toolbox. In the Supplementary Material, we report the full methods and provide additional plots. 
bioRxiv preprint doi: https://doi.org/10.1101/2021.06.09.447763; this version posted August 12, 2021. The copyright holder for this preprint (which was not certified by peer review) is the author/funder, who has granted bioRxiv a license to display the preprint in perpetuity. It is made available under aCC-BY-ND 4.0 International license.

changes in the fidelity of the neural signature. We use this logic to test brain time warping: if the oscillations of interest really do clock cognition, then retuning data based on their dynamics should bring out fluctuating patterns across the classifier's performance over time (periodicity). This approach to analysing dynamic cognition in the brain is implemented in the Brain Time Toolbox, but brain time warped data may also be used outside the toolbox.

The first dataset is a simulation of a basic attentional spotlight model with dipoles oscillating at an alpha frequency. We generated two classes of data with separate dipole parameters (Figure 3A), mimicking covert attention to the left (class 1) or right hemifield (class 2). We classified the data using linear discriminant analysis (LDA). In the clock time data, the random starting phases and frequency drift preclude a readout of the ground truth pattern (Figure 3C; top row). After brain time warping, the temporal generalization matrix (TGM) contains a statistically robust periodic pattern at the warped frequency, indicating brain time warping has unveiled the fluctuating neural signature (Figure 3C; bottom row).

In a second dataset, we studied spatial navigation in rodents by analysing grid cells in the entorhinal cortex (data obtained from [34]). Local field potentials (LFP) and single units were measured while rodents navigated through a rectangular open field (Figure 4A). Trials were split into two conditions depending on whether the animal was travelling through a field coded by the grid cell or through another location not coded by the grid cell (both centred to time $=0$ ). We trained
Rodent

A.

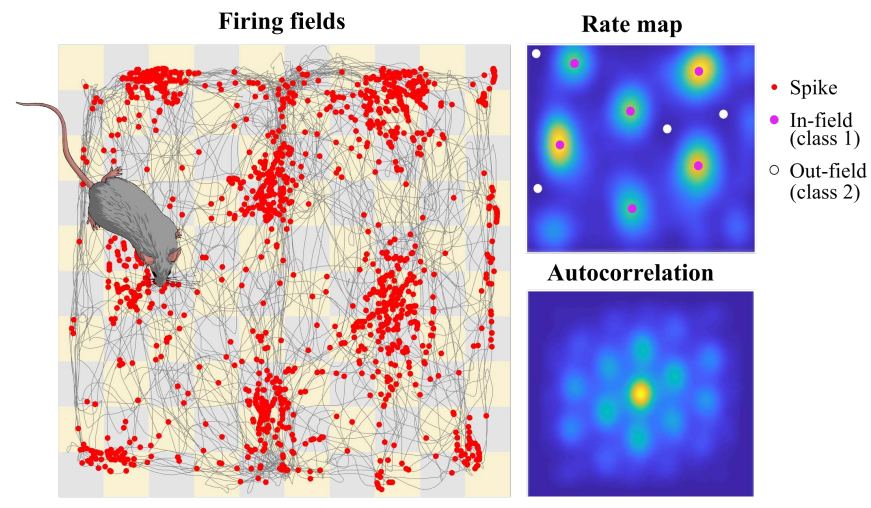

Human

EEG
B.
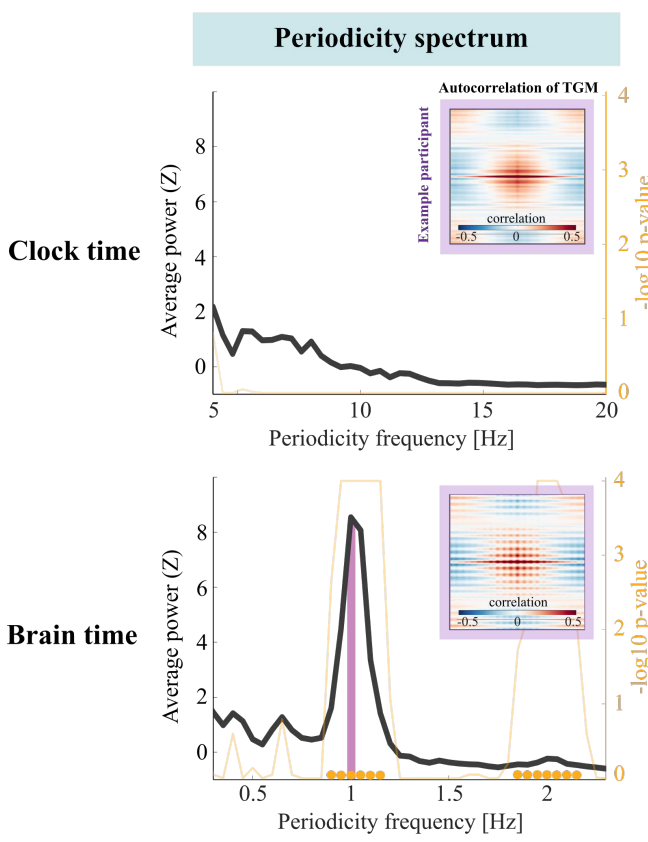

C.
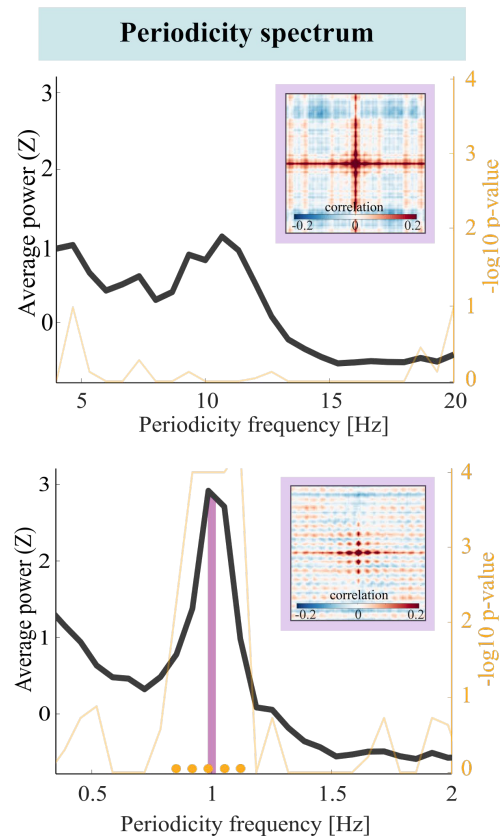

D.
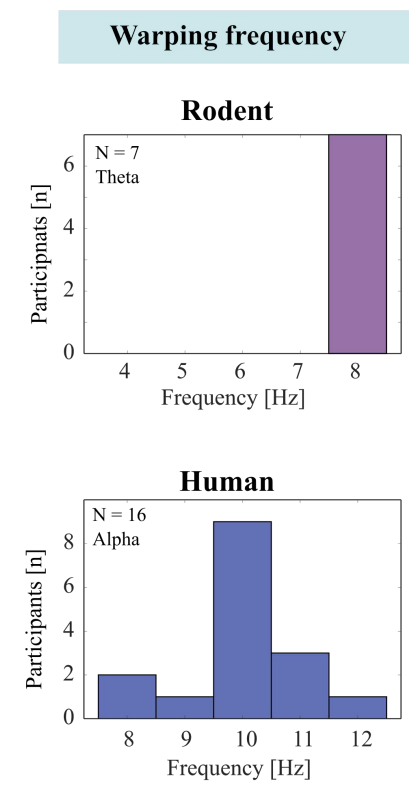

Figure 4: Brain time warping reveals periodic patterns in rodents and humans

(A) (Left) While rodents navigated through an open field, single- and multi-unit recordings were obtained. We display cell spike locations in the field, its smoothed representation (rate map), and this representation's autocorrelation. We cut the data depending on whether the animal was traveling through a location coded by the grid cell (class 1) or not (class 2) and brain time warped the data to theta oscillations (4 to $8 \mathrm{~Hz}$ ) obtained from local field potential (LFP) recordings in the entorhinal cortex. Next, we decoded grid cell activity (across $\mathrm{N}=7$ sessions) and tested brain time warping. (Right) In a human dataset, electroencephalography (EEG) was recorded while participants $(\mathrm{N}=16)$ viewed random dot kinematograms, with dots moving at one of two levels of coherence $(25.6 \%$ or $51.2 \%)$ in a direction ranging from $1^{\circ}$ to $360^{\circ}$. We pooled coherence and binarized direction toward left- (class 1 ) or rightward (class 2 ) motion conditions. Next, we brain time warped to parietal alpha oscillations [8] and tested for brain time warping's effects. (B) Warping the rodent data to LFP theta unveils statistically robust periodicity around the warping frequency and around its first harmonic $(\mathrm{p}<0.001)$, while the clock time spectrum shows no significant peaks. The inset shows an example session's autocorrelation map of the TGM (C) In the human dataset, brain time warping reveals periodic patterns around the warping frequency ( $\mathrm{p}<$ 0.001 ), indicating the neural signature of attention fluctuates during motion perception. (D) Summary statistics of the warping frequency across participant for each analysis. (General) Most of the general notes of Figure 3 apply to this figure too. In the Supplementary Material, we report the full methods and provide additional plots. These plots include TGMs, autocorrelation maps, grid cell maps, spike-field coupling between theta and grid cell spikes, and so forth. 
bioRxiv preprint doi: https://doi.org/10.1101/2021.06.09.447763; this version posted August 12, 2021. The copyright holder for this preprint

(which was not certified by peer review) is the author/funder, who has granted bioRxiv a license to display the preprint in perpetuity. It is made available under aCC-BY-ND 4.0 International license.

a classifier to differentiate the in-field (class 1) and out-field conditions (class 2) based on the firing rate of grid cells. The clock time data shows high classifier performance at time $=0$, which is unsurprising considering this is where we defined a difference between classes. However, surprisingly, no periodicity is evident over time (Figure 4B; top) despite grid cells strongly phase locking to theta oscillations ([35]; Supplementary Material 3.2.1). Importantly, brain time warping the firing rates of grid cells to theta oscillations obtained from the LFP recovers this periodicity (Figure 4B; bottom).

In a third dataset, we decoded motion direction using EEG data recorded while participants viewed moving dots (Figure 4A, right; data obtained from [36]). We predicted that evidence for spatial attention would rhythmically fluctuate at participants' individual alpha frequency. Again, we tested for periodic patterns in a TGM obtained from a classifier, this time trained on left- (class 1) and rightward (class 2) motion conditions. Brain time warping reveals periodicity at participants' individual alpha frequency which was not significant in the original clock time data (Figure 4C). In the Supplementary Material (1.0), we report additional details on each analysis.

\section{When is retuning clock and brain time necessary?}

The need to use brain time warping or other methods to retune depends on (1) the degree to which clock and brain time are in disharmony, and (2) the degree to which such disharmony interferes with analyses. Below, we elaborate on both criteria.

The degree to which disharmony is present depends on the levels of processing involved in the employed experimental paradigm. Take once again the spatial attention study. Between the moving dots' light hitting the participant's retina and their pressing of the button to indicate motion direction, a vast amount of subcortical and cortical processing transpires. While internal dynamics start to coordinate stimulus processing as early as the thalamus [37,38], or even earlier [39], disharmony is most prevalent in higher level regions - where processes such as attention and decision-making operate in full swing. This is because at this late stage, information has passed through many cell ensembles where different oscillations have each exerted a temporal footprint. As these footprints add up, clock time falls increasingly out of tune with brain time. As a result, we lose track of brain dynamics and the information it provides about cognition, such as whether dots make it to awareness [40] or whether features of the dots (such as their colour) are available to working memory [41]. Thus, there is a gradation in clock time's distorting effect which depends on the extent of cortical processing. Research that restricts analyses to subcortical processing or very early evoked potentials benefits comparatively little from retuning, while retuning may be a vital step to understand brain patterns in late stages - with at least one exception.

Retuning is unnecessary for analyses that do not rely on temporal variations of the neural signature. For example, a researcher may want to study the trial-average activity in parietal regions as a function of the proportion of dots that moved, or map aggregate differences in network connectivity between participants. Here it is true as ever that brain dynamics play their part in the subprocesses involved, but the analyses are insensitive to time variations, leaving them unafflicted by oscillations' footprint.

In short, retuning clock and brain time is necessary depending on the degree to which both the mechanisms of study and the employed analyses depend on the brain's internal dynamics. On the whole, few electrophysiology studies are exempt from clock time's distorting effects.

\section{Is retuning using brain time warping circular?}

A potential concern with brain time warping could be that it trivially imposes oscillatory structure onto the data. The worry here is that the algorithm makes the data fluctuate even when warping to oscillations that do not clock cognition. In this section we argue when the algorithm is on safe grounds, and when vigilance is needed.

First, as a note of clarification, the path used for brain time warping does not contain oscillatory structure. That is to say, if the path were applied to a random time series, it would not mould it to increase its oscillatory shape. This is because the path is instead designed to show when the oscillation used for warping undergoes nonstationary behaviour. Nevertheless, an important concern remains. Brain time warping applies the path to the data in order to align it with brain dynamics, so if the warping signal is within the data, its stationarity will increase introducing some oscillatory structure after all.

Circularity concerns can easily be avoided by ensuring independence between the warping source and transformed data. If the cause of circularity resides in changes to the warping signal, then removing it from the transformed data obviates circularity concerns. We report suggestions on how to achieve data independence for each electrophysiology method, describing how they can be implemented before or after warping (Supplementary Material 2.4.1).

What if independence between warping source and data is impossible? In this case, testing for temporal patterns using the Brain Time Toolbox is safe as its method to obtain null distributions benefits equally from potential circularity as the empirical distribution. However, when analysing warped data with dependence outside the toolbox, it is important to determine on a case-by-case basis whether circularity is a concern based on the planned analyses at hand. To aid this process, the toolbox tracks dependence between warping source and warped data and raises a warning when circularity could be an issue.

Besides these methodological arguments, we also show that brain time warping does not trivially impose oscillatory structure by warping to a control frequency [42] in the simulated dataset, where we can objectively determine when a false positive result happens. This specificity analysis showed no statistically significant periodicity at the warped control frequency (Supplementary Material 1.2.2).

With these points in mind, we nevertheless emphasize that brain time warping is a hypothesis-driven method that capitalizes on the temporally coordinating nature of oscillations. Hence, we recommend warping only using oscillations predicted to fit that bill to avoid false positive results by chance. To enable hypothesis-driven warping, the Brain Time Toolbox computes a variety of information about warping signals including their time frequency characteristics, waveshape, and topographical profile - allowing users to make informed decisions about which signal they wish to designate as brain time.

\section{Other ways to account for disharmony}

The thrust of our argument is that we should factor in brain dynamics in neuroscientific analysis. Retuning clock and brain time using brain time warping offers a comprehensive way of doing so. But many other methods are possible, which range in scope. At minimum, the phase of clocking oscillations can be used to explain variance in measures of interest, such as behaviour [43] or classifier performance [19]. More extensive approaches include locking analyses to oscillatory peaks or troughs [44], linear warping algorithms [45], and re-sorting data with phase as the time axis. Interestingly, the latter method has been used to demonstrate phase precession in humans, results 
bioRxiv preprint doi: https://doi.org/10.1101/2021.06.09.447763; this version posted August 12, 2021. The copyright holder for this preprint (which was not certified by peer review) is the author/funder, who has granted bioRxiv a license to display the preprint in perpetuity. It is made available under aCC-BY-ND 4.0 International license.

which were absent when the analysis was performed in clock time [46].

\section{Brain time is not unitary}

The phrase "brain time" is used to emphasize the conceptual departure from a format extrinsic to the brain. We do not mean to suggest that there is a single brain time. Rather, different cognitive processes are clocked by different groups of oscillations, each with their own frequency and source. In this sense, brain time is analogous to a concept like "Earth time", which contrasts itself with time on other planets whilst further decomposing into different time zones. Finally, brain time in the present context does not refer to timekeeping in the brain. Instead, it refers to the oscillatory dynamics by which the brain coordinates cognition generally, of which temporal cognition is a specific instance [47].

\section{Conclusion}

Where does this leave us? We believe that rather than imposing a foreign unit of time onto the brain while studying its function, the brain is best understood as a system with its own dynamics and temporal organization. Upon inspection, the brain operates rhythmically, with brain oscillations as a key player. This has important consequences for scientific analysis. If it is true that oscillations clock brain activity to coordinate cognition, then their dynamics should heavily inform how we study evolving data patterns. In contrast, analysing such patterns in the default clock time format is likely to yield a distorted readout as the brain's internal dynamics do not scale linearly to sequences of (milli)seconds. We introduce brain time warping as a new method to account for disharmony in brain data, dynamically transforming electrophysiology data structures in a way that brings them in line with brain dynamics. The Brain Time Toolbox implements brain time warping, facilitating analysis on the oscillatory dynamics of the cognitive brain.

\section{Acknowledgments}

We thank Ehren Newman, Federica Meconi, Gi-Yeul Bae, and Steven J. Luck for sharing their data, and Benjamin Griffiths for his insightful comments on the Brain Time Toolbox.

\section{References}

[1] Antle, M. C., \& Silver, R. (2005). Orchestrating time: arrangements of the brain circadian clock. Trends in Neurosciences, 28(3), 145-151. https://doi.org/https://doi.org/10.1016/j.tins.2005.01.003

[2] MacDonald, C. J., Lepage, K. Q., Eden, U. T., \& Eichenbaum, H. (2011). Hippocampal "Time Cells" Bridge the Gap in Memory for Discontiguous Events. Neuron, 71(4), 737-749. https://doi.org/https://doi.org/10.1016/j.neuron.2011.07.012

[3] Llinás, R. R. (1988). The intrinsic electrophysiological properties of mammalian neurons: insights into central nervous system function. Science (New York, N.Y.), 242(4886), 1654-1664. https://doi.org/10.1126/science.3059497

[4] Buzsáki, G., \& Draguhn, A. (2004). Neuronal oscillations in cortical networks. Science (New York, N.Y.), 304(5679), 1926-1929. https://doi.org/10.1126/science.1099745

[5] Engel, A. K., Fries, P., \& Singer, W. (2001). Dynamic predictions: Oscillations and synchrony in top-down processing. Nature Reviews Neuroscience, 2(10), 704-716. https://doi.org/10.1038/35094565

[6] Jensen, O., \& Mazaheri, A. (2010). Shaping functional architecture by oscillatory alpha activity: gating by inhibition. Frontiers in Human Neuroscience, 4, 186. https://doi.org/10.3389/fnhum.2010.00186

[7] Klimesch, W. (2012). $\alpha$-band oscillations, attention, and controlled access to stored information. Trends in Cognitive Sciences, 16(12), 606-617. https://doi.org/10.1016/j.tics.2012.10.007

[8] Capotosto, P., Babiloni, C., Romani, G. L., \& Corbetta, M. (2009). Frontoparietal cortex controls spatial attention through modulation of anticipatory alpha rhythms. The Journal of Neuroscience: The Official Journal of the Society for Neuroscience, 29(18), 5863-5872. https://doi.org/10.1523/JNEUROSCI.0539-09.2009

[9] Luo, H., \& Poeppel, D. (2012). Cortical Oscillations in Auditory Perception and Speech: Evidence for Two Temporal Windows in
Human Auditory Cortex. In Frontiers in Psychology (Vol. 3, p. 170). https:/www.frontiersin.org/article/10.3389/fpsyg.2012.00170

[10] Lakatos, P., Shah, A. S., Knuth, K. H., Ulbert, I., Karmos, G., \& Schroeder, C. E. (2005). An oscillatory hierarchy controlling neuronal excitability and stimulus processing in the auditory cortex. Journal of Neurophysiology, 94(3), 1904-1911. https://doi.org/10.1152/jn.00263.2005

[11] Busch, N. A., Dubois, J., \& VanRullen, R. (2009). The phase of ongoing EEG oscillations predicts visual perception. The Journal of Neuroscience: The Official Journal of the Society for Neuroscience, 29(24), 7869-7876. https://doi.org/10.1523/JNEUROSCI.011309.2009

[12] Romei, V., Gross, J., \& Thut, G. (2010). On the Role of Prestimulus Alpha Rhythms over Occipito-Parietal Areas in Visual Input Regulation: Correlation or Causation? The Journal of Neuroscience, 30(25), 8692 LP - 8697. https://doi.org/10.1523/JNEUROSCI.016010.2010

[13] Ai, L., \& Ro, T. (2013). The phase of prestimulus alpha oscillations affects tactile perception. Journal of Neurophysiology, 111(6), 1300 1307. https://doi.org/10.1152/jn.00125.2013

[14] Ruzzoli, M., \& Soto-Faraco, S. (2014). Alpha Stimulation of the Human Parietal Cortex Attunes Tactile Perception to External Space. Current Biology, 24(3), 329-332. https://doi.org/https://doi.org/10.1016/j.cub.2013.12.029

[15] Nowak, M., Zich, C., \& Stagg, C. J. (2018). Motor Cortical Gamma Oscillations: What Have We Learnt and Where Are We Headed? Current Behavioral Neuroscience Reports, 5(2), 136-142. https://doi.org/10.1007/s40473-018-0151-z

[16] Joundi, R. A., Jenkinson, N., Brittain, J.-S., Aziz, T. Z., \& Brown, P. (2012). Driving Oscillatory Activity in the Human Cortex Enhances Motor Performance. Current Biology, 22(5), 403-407. https://doi.org/https://doi.org/10.1016/j.cub.2012.01.024

[17] Hasselmo, M. E., Bodelón, C., \& Wyble, B. P. (2002). A proposed function for hippocampal theta rhythm: separate phases of encoding and retrieval enhance reversal of prior learning. Neural Computation, 14(4), 793-817. https://doi.org/10.1162/089976602317318965

[18] Hanslmayr, S., Axmacher, N., \& Inman, C. S. (2019). Modulating Human Memory via Entrainment of Brain Oscillations. Trends in Neurosciences, 42(7), 485-499. https://doi.org/10.1016/j.tins.2019.04.004

[19] Kerrén, C., Linde-Domingo, J., Hanslmayr, S., \& Wimber, M. (2018). An Optimal Oscillatory Phase for Pattern Reactivation during Memory Retrieval. Current Biology, 28(21), 3383-3392.e6. https://doi.org/https://doi.org/10.1016/j.cub.2018.08.065

[20] Jacobs, J., Hwang, G., Curran, T., \& Kahana, M. J. (2006). EEG oscillations and recognition memory: Theta correlates of memory retrieval and decision making. NeuroImage, 32(2), 978-987. https://doi.org/https://doi.org/10.1016/j.neuroimage.2006.02.018

[21] Polanía, R., Moisa, M., Opitz, A., Grueschow, M., \& Ruff, C. C. (2015). The precision of value-based choices depends causally on frontoparietal phase coupling. Nature Communications, 6(1), 8090. https://doi.org/10.1038/ncomms9090

[22] Mazaheri, A., \& Jensen, O. (2006). Posterior $\alpha$ activity is not phase-reset by visual stimuli. Proceedings of the National Academy of Sciences of the United States of America, 103(8), 2948 LP - 2952. https://doi.org/10.1073/pnas.0505785103

[23] Jansen, B. H., Agarwal, G., Hegde, A., \& Boutros, N. N. (2003). Phase synchronization of the ongoing EEG and auditory EP generation. Clinical Neurophysiology, 114(1), 79-85. https://doi.org/https://doi.org/10.1016/S1388-2457(02)00327-9

[24] Cole, S. R., \& Voytek, B. (2017). Brain Oscillations and the Importance of Waveform Shape. Trends in Cognitive Sciences, 21(2), 137-149. https://doi.org/10.1016/j.tics.2016.12.008

[25] Sauseng, P., Klimesch, W., Stadler, W., Schabus, M., Doppelmayr, M., Hanslmayr, S., Gruber, W. R., \& Birbaumer, N. (2005). A shift of visual spatial attention is selectively associated with human EEG alpha activity. The European Journal of Neuroscience, 22(11), 2917 2926. https://doi.org/10.1111/j.1460-9568.2005.04482.x

[26] Capotosto, P., Babiloni, C., Romani, G. L., \& Corbetta, M. (2009). Frontoparietal cortex controls spatial attention through modulation of anticipatory alpha rhythms. The Journal of Neuroscience: The Official Journal of the Society for Neuroscience, 29(18), 5863-5872. https://doi.org/10.1523/JNEUROSCI.0539-09.2009

[27] Buzsáki G. (2019) The brain from inside out. New York: Oxford University Press.

[28] Scharnowski, F., Rees, G., \& Walsh, V. (2013). Time and the brain: neurorelativity: The chronoarchitecture of the brain from the neuronal rather than the observer's perspective. Trends in Cognitive Sciences, 17(2), 51-52. https://doi.org/10.1016/j.tics.2012.12.005

[29] Kostov, V. B., McCullough, P. R., Carter, J. A., Deleuil, M., Díaz, R. F., Fabrycky, D. C., Hebrard, G., Hinse, T. C., Mazeh, T., \& Orosz, J. A. (2014). Kepler-413b: a slightly misaligned, Neptune-size transiting circumbinary planet. The Astrophysical Journal, 784(1), 14. 
bioRxiv preprint doi: https://doi.org/10.1101/2021.06.09.447763; this version posted August 12, 2021. The copyright holder for this preprint (which was not certified by peer review) is the author/funder, who has granted bioRxiv a license to display the preprint in perpetuity. It is made available under aCC-BY-ND 4.0 International license.

[30] Klimesch, W. (1999). EEG alpha and theta oscillations reflect cognitive and memory performance: a review and analysis. Brain Research Reviews, 29(2), 169-195. https://doi.org/https://doi.org/10.1016/S0165-0173(98)00056-3

[31] Sakoe, H., \& Chiba, S. (1978). Dynamic programming algorithm optimization for spoken word recognition. IEEE Transactions on Acoustics, Speech, and Signal Processing, 26(1), 43-49. https://doi.org/10.1109/TASSP.1978.1163055

[32] Berndt, D. J., \& Clifford, J. (1994). Using Dynamic Time Warping to Find Patterns in Time Series. Proceedings of the 3rd International Conference on Knowledge Discovery and Data Mining, 359-370.

[33] Benjamini, Y., \& Hochberg, Y. (1995). Controlling the False Discovery Rate: A Practical and Powerful Approach to Multiple Testing. Journal of the Royal Statistical Society: Series B (Methodological), 57(1), 289300. https://doi.org/https://doi.org/10.1111/j.25176161.1995.tb02031.x

[34] Newman, E. L., \& Hasselmo, M. E. (2014). Grid cell firing properties vary as a function of theta phase locking preferences in the rat medial entorhinal cortex. In Frontiers in Systems Neuroscience (Vol. 8, p. 193). https://www.frontiersin.org/article/10.3389/fnsys.2014.00193

[35] Burgess, N., \& O'Keefe, J. (2011). Models of place and grid cell firing and theta rhythmicity. Current Opinion in Neurobiology, 21(5), 734 744. https://doi.org/10.1016/j.conb.2011.07.002

[36] Bae, G.-Y., \& Luck, S. J. (2019). Decoding motion direction using the topography of sustained ERPs and alpha oscillations. NeuroImage, 184, 242-255. https://doi.org/10.1016/j.neuroimage.2018.09.029

[37] Hughes, S. W., Lörincz, M., Cope, D. W., Blethyn, K. L., Kékesi, K. A., Parri, H. R., Juhász, G., \& Crunelli, V. (2004). Synchronized Oscillations at $\alpha$ and $\theta$ Frequencies in the Lateral Geniculate Nucleus. Neuron, 42(2), 253-268. https://doi.org/https://doi.org/10.1016/S08966273(04)00191-6

[38] Vijayan, S., \& Kopell, N. J. (2012). Thalamic model of awake alpha oscillations and implications for stimulus processing. Proceedings of the National Academy of Sciences, 109(45), 18553 LP - 18558. https://doi.org/10.1073/pnas.1215385109

[39] Koepsell, K., Wang, X., Vaingankar, V., Wei, Y., Wang, Q., Rathbun, D. L., Usrey, W. M., Hirsch, J. A., \& Sommer, F. T. (2009). Retinal oscillations carry visual information to cortex. Frontiers in Systems Neuroscience, 3, 4. https://doi.org/10.3389/neuro.06.004.2009
[40] VanRullen, R. (2016). Perceptual Cycles. Trends in Cognitive Sciences, 20(10), 723-735.

https://doi.org/https://doi.org/10.1016/j.tics.2016.07.006

[41] ten Oever, S., De Weerd, P., \& Sack, A. T. (2020). Phase-dependent amplification of working memory content and performance. Nature Communications, 11(1), 1832. https://doi.org/10.1038/s41467-02015629-7

[42] Pletzer, B., Kerschbaum, H., \& Klimesch, W. (2010). When frequencies never synchronize: the golden mean and the resting EEG. Brain Research, 1335, 91-102. https://doi.org/10.1016/j.brainres.2010.03.074

[43] Kösem, A., Gramfort, A., \& van Wassenhove, V. (2014). Encoding of event timing in the phase of neural oscillations. NeuroImage, 92, 274 284. https://doi.org/https://doi.org/10.1016/j.neuroimage.2014.02.010

[44] Bonnefond, M., \& Jensen, O. (2015). Gamma Activity Coupled to Alpha Phase as a Mechanism for Top-Down Controlled Gating. PLOS ONE, 10(6), e0128667. https://doi.org/10.1371/journal.pone.0128667

[45] Williams, A. H., Poole, B., Maheswaranathan, N., Dhawale, A. K., Fisher, T., Wilson, C. D., Brann, D. H., Trautmann, E. M., Ryu, S., Shusterman, R., Rinberg, D., Ölveczky, B. P., Shenoy, K. V, \& Ganguli, S. (2020). Discovering Precise Temporal Patterns in LargeScale Neural Recordings through Robust and Interpretable Time Warping. Neuron, 105(2), 246-259.e8. https://doi.org/10.1016/j.neuron.2019.10.020

[46] Qasim, S. E., Fried, I., \& Jacobs, J. (2021). Phase precession in the human hippocampus and entorhinal cortex. Cell. https://doi.org/10.1016/j.cell.2021.04.017

[47] van Wassenhove, V. (2016). Temporal cognition and neural oscillations. Current Opinion in Behavioral Sciences, 8, 124-130. https://doi.org/10.1016/j.cobeha.2016.02.012 


\section{Supplementary Material}

Click on section numbers in the table of contents or text to jump to a section.

\section{Table of contents}

\section{Dataset analysis}

1.1 General methods

1.2 Simulation

1.2.1 Methods

1.2.2 Control analysis

1.3 Rodent

1.4 Human

$$
\text { 1.3.1 Methods }
$$

1.4.1 Methods

\section{Toolbox methods}

2.1 Introduction

2.1.1 Requirements

2.1.2 Download and installation

2.2 Operation 1: Brain time warping

2.2.1 Obtaining warping sources

2.2.2 Analysing warping sources

2.2.3 Selecting a warping signal

2.2.4 Warping clock to brain time

2.3 Operation 2 : Testing periodicity

2.3.1 Classifying the data

2.3.2 Quantifying classifier periodicity

2.3.3 First-level statistics of periodicity

2.3.4 Second-level statistics of periodicity

2.4 Methodological considerations

2.4.1 Circularity concerns

2.4.2 Warping artefacts

\section{Supplementary data plots}

\subsection{Simulation}

3.1.1 Classification (main analysis)

3.1.2 Classification (control analysis)

3.2 Rodent

3.2.1 Grid cells

3.2.2 Spike-field coupling

3.2.3 Classification

3.3 Human

3.3.1 Classification

\section{Supplementary references}




\section{Data analysis}

\subsection{General methods}

We tested whether brain time warping increases dynamic patterns of cognition by applying the Brain Time Toolbox (abbreviated braintime) to three datasets. We divided each dataset into two classes, with their difference in brain activity predicted to reflect the neural signature of cognition (e.g., the difference in activity between left and right cued trials reflects spatial attention). We tested whether brain time warping increases periodic fluctuations in the neural signature. Insofar the oscillation used for warping temporally coordinates a studied cognitive process, transforming data according to its dynamics should enhance such fluctuations. We tested this prediction by applying a linear discriminant analysis (LDA) and analysing its performance over time for brain time warped data. Specifically, we generated temporal generalization matrices (TGMs) for each participant and applied a Fast Fourier Transform (FFT) over each of its rows and columns. We refer to this procedure as a "periodicity analysis". For extensive details on periodicity analysis (as well as details on braintime terminology and all other analytical steps), we refer to braintime's methods section (2.1 to 2.4). In this section, we first describe information common to the analysis of each dataset, before discussing them individually.

\section{Classification}

We compare second-level periodicity results between clock time data (input to the toolbox) and brain time data (output of the toolbox). To do so, we trained an LDA with 5 folds and 10 repetitions on the data and obtained 50 empirical TGMs and a null distribution of 50 permuted TGMs (obtained by class label shuffling, 2.3.3). Classifier performance was defined in terms of accuracy (i.e., the proportion of correctly classified trials). We used braintime to quantify periodicity in the TGMs, which yielded $n$ empirical periodicity spectra and $50 \times n$ permuted spectra, where $n$ is the number of participants of each dataset. To obtain second-level results, we set braintime to obtain $10^{6}$ permuted power values for each periodicity frequency by randomly grabbing (with replacement) from the total pool of permuted TGMs, from which p-values are calculated (2.3.4).

\section{Cluster correction}

We established whether the classifier was able to differentiate both classes of data above chance using cluster correction in MVPA Light (2.3.4). We use $10^{4}$ permutations to test for significant clusters.

\section{Warping source extraction}

For the simulation and human analyses, we use independent component analysis (ICA) to decompose the input clock time data into additive subcomponents with statistically independent characteristics. Each component served as a warping source, meaning each contained a potential warping signal that could be designated as brain time. After brain time warping, we removed the warping source (ICA component) that was used for warping from the data before continuing to periodicity analyses. This removes the warping signal from the output data, introducing data independence (2.4.1).

For the rodent analysis, we warp using local field potential channels, and introduce independence by removing the selected channel from the data, leaving only the warped single-unit data.

\section{Brain Time Toolbox settings}

We used braintime's default consistenttime method to ensure the brain time warped data restricts itself to the specified time window for each analysis (2.4.2). To obtain time frequency information of each warping source, we applied a wavelet analysis (fitting 5 cycles to the data). To correct for multiple comparisons across the tested frequencies in the periodicity spectra, we adjusted p-values for the false discovery rate (FDR; Benjamini, \& Hochberg, 1995).

\subsection{Simulation}

We simulated a basic attentional spotlight model (main Figure 3) with dipoles oscillating at an alpha frequency ( 8 to $12 \mathrm{~Hz}$ ). To introduce disharmony between clock and brain time, we introduced variable starting phases and frequency drift in the primary dipoles. We predicted that brain time warping would selectively increase periodicity at the ground truth frequency by adjusting for nonstationarities in the warping signal. Braintime contains the MATLAB (The MathWorks, Natick, Massachusetts, USA) code used to generate the simulated 
dataset, which includes options to change simulation parameters (such as the degree of frequency drift, pink noise amplitude, the number and duration of trials, and so forth).

\subsubsection{Methods}

We used FieldTrip's (Oostenveld et al., 2011) ft_dipolesimulation function to generate an electrophysiology dataset where the ground truth consists in a periodic fluctuation of covert attention at alpha frequencies. We simulated $n=10$ datasets with 120 trials and two conditions (covert attention to the left and right hemifield; LHF and RHF). Each trial lasted one second and had a sampling rate of $200 \mathrm{Hertz}$ (Hz). Across both conditions and all participants, we held constant the orientation and location of the primary dipoles that instantiated the neural signature of attention. The remaining parameters were randomized, such as the frequency, location, and orientation of non-primary dipoles ("random" dipoles), as well as each dipole's starting phase. We modelled 3 primary dipoles, and 8 random dipoles. The primary dipoles contained different parameters across the two classes of data. In contrast, the random dipoles contained parameters that were uncorrelated to each class, yielding no meaningful average difference in neural activity between conditions. In this simulation, our primary focus was to create a dataset suitable to test brain time warping (with a neural signature and nonstationarity) rather than to accurately model attention in close accordance with theoretical models.

\section{Primary dipole configuration}

We placed the conductor dipole in the right parietal cortex with an amplitude of 1 (in arbitrary units), oscillating at a random alpha frequency. This conductor dipole "conducted" the phase of two follower dipoles, one located in each visual cortex, oscillating at the same frequency and oriented with the same $\mathrm{x}, \mathrm{y}$, and $\mathrm{z}$ coordinates. We generated two classes: 60 trials of LHF and RHF data. The two conditions contained a difference in parameters and thereby brain activity - that the classifier could exploit to differentiate the data and obtain the ground truth signature of attention. Specifically, for both conditions, we changed two parameters:

- The relative amplitude between follower dipoles. The follower dipole contralateral to the hemifield condition oscillated at half the amplitude (0.5) compared to the ipsilateral dipole (1) - mimicking contralateral inhibition of alpha.

- Phase relation between primary dipoles. The conductor dipole oscillated in antiphase with the follower dipole contralateral to the hemifield. In contrast, it oscillated in phase with the follower dipole ipsilateral to the hemifield (barring a $\frac{\pi}{6}$ phase delay to mimic signal conductance delay).

In addition to these condition-specific differences, each primary dipole contained 1/F noise, variable starting phase, and frequency drift. We implemented frequency drift through a random walk approach, where the frequency of all three primary dipoles decreased or increased by $0.05 \mathrm{~Hz}$ per sample.

\section{Random dipole configuration}

To decrease the signal to noise ratio of the neural signature exerted by primary dipoles, we introduced 8 random dipoles that oscillated at random frequencies, limited to $2 \mathrm{~Hz}$ above and below the primary dipole's frequency to avoid interference with the neural signature. These dipoles oscillated at an amplitude of 1, contained 1/F noise, and were placed randomly across the brain with a random orientation.

\section{Brain time warping}

We considered the resulting datasets as separate participants with their own alpha frequency. We used ICA to obtain warping sources and warped each participant to a source with (1) a topographical activity profile around occipital and parietal regions, (2) and a high amplitude alpha peak. We found that brain time warping unveils periodicity at the warping frequency which was absent in clock time. This demonstrates that the variable starting phases and frequency drift preclude the classifier from detecting reliable differences in neural activity between both classes of data. However, after brain time warping has been applied, the nonstationary dynamics of the primary dipoles are accounted for, resulting in a significant effect at the warping frequency $(\mathrm{p}=<0.005$; main Figure 3). We provide TGMs, autocorrelation maps and first-level periodicity spectra in 3.1.1.

\subsubsection{Control analysis}

To test for the specificity of brain time warping, we warped to the golden mean of each participant's warping frequency as obtained in the main analysis. The logic is that if brain time warping is non-specific and prone to false positive results by trivially imposing oscillatory structure, then it should do so even for frequencies predicted to not have a clocking role in cognition. To test this possibility, we repeated the main analysis by warping to the 
golden mean of each participant's warping frequency $(\approx$ warping frequency $\times 1.618)$. We chose the golden mean as our control frequency because, at this frequency, the warping signal (which is predicted to be the clocking oscillation) is least expected to synchronize to different oscillations (Pletzer, Kerschbaum \& Klimesch, 2010). The control analysis changed the warping frequencies from alpha $(8$ to $12 \mathrm{~Hz})$ to approximately $12.9 \mathrm{~Hz}$ to 19.4 $\mathrm{Hz}$. All other parameters were unchanged from the main analysis. The control analysis does not show significant periodicity at any frequency. We provide TGMs, autocorrelation maps and first-level periodicity spectra in 3.1.2.

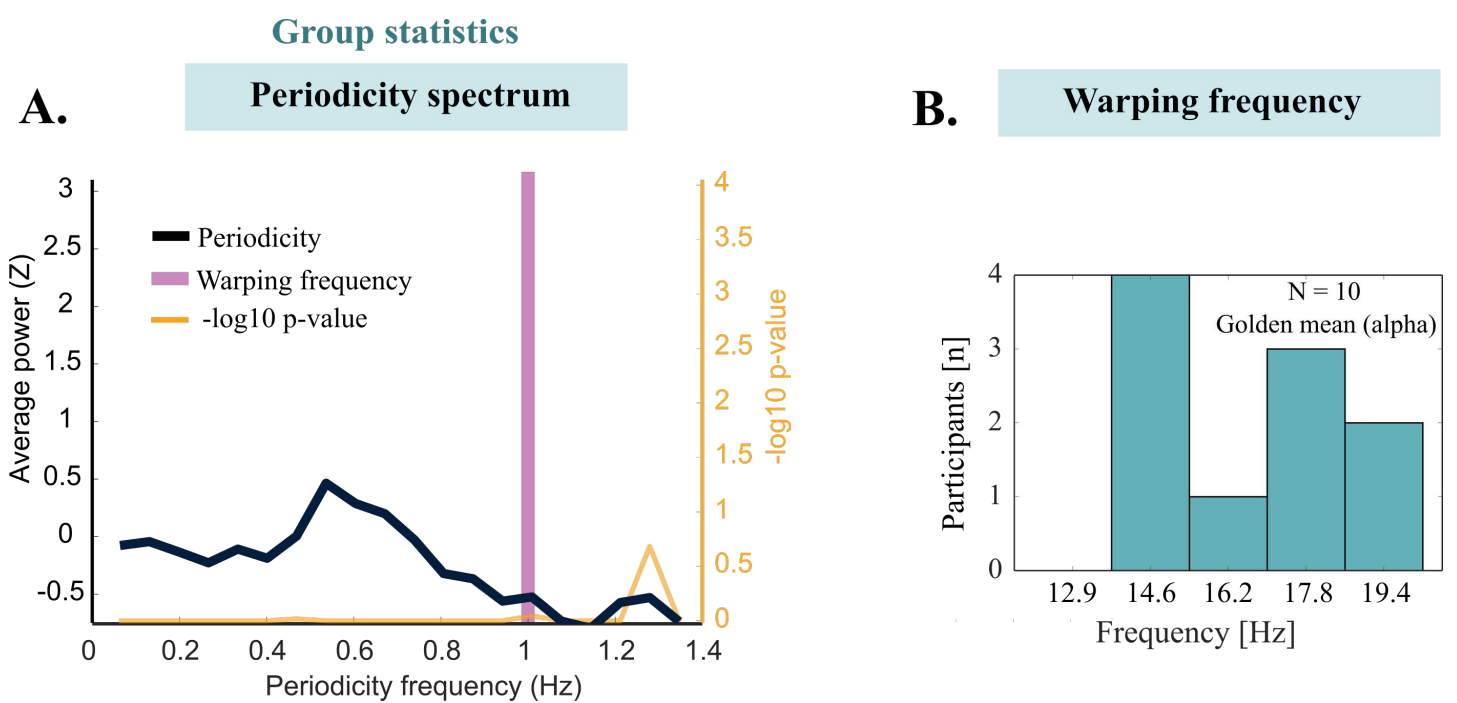

Supplementary Figure 1: Second-level periodicity for the control analysis of the simulation dataset

(A) We found no significant periodicity when warping to the golden mean of each participant's alpha oscillation. (B) Summary statistics of the warping frequency across participant.

\subsection{Rodent}

We brain time warped a rodent dataset obtained from Newman \& Hasselmo (2014). In this study, local field potentials (LFP) and single units were measured from the entorhinal cortex of Long-Evans rats as they navigated through a rectangular open field. In the following section, we describe how we reformatted and analysed the data to test the effects of brain time warping. For methodological details on the recordings such as data acquisition, surgical procedures, and behavioural protocols, we refer to Newman \& Hasselmo (2014). We hypothesized that brain time warping would increase the periodicity with which a classifier is able to differentiate when an animal is inside or outside the firing field of a grid cell. To this end, we brain time warped grid cell activity to theta oscillations in the LFP. The logic is that if theta clocks grid cell activity, then periodic patterns in their spiking activity should be enhanced after brain time warping (through its correcting of nonstationarities in brain time).

\subsubsection{Methods}

Primary grid cells

We inspected single cell recordings from 7 recording sessions ( $\mathrm{N}=2$ rodents) and extracted one primary grid cell for each recording, which we used to define the in- and outfield condition. We selected a primary grid cell by comparing each cell's firing rate map for triangular structure and the periodicity of these maps using a 2D autocorrelation analysis (Hafting, Fyhn, Molden, Moser \& Moser, 2005). In 3.2.1, we display the smoothed firing rate maps of each session's primary grid cell and how we devised classes based on their firing fields.

\section{Spike-field coupling}

Brain time warping is only expected to enhance periodicity if grid cells phase lock to theta. Thus, we verified spike-field coupling for selected grid cells. In 3.2.2, we show the spike-field coupling of one example grid cell. 


\section{Data preparation}

For each session, we created a separate structure for the spike activity of grid cells (between 2 and 4 channels; convolved with a Gaussian) and LFP recordings (2 channels). We split each structure into two classes of data based on when rodents were inside a location coded by the primary grid cell's firing field (defined by local maxima in the smoothed firing rate map) or outside of it (defined by local minima). As a consequence of this split, the data at $\mathrm{t}=0$ reflected the animal's presence in a firing field (class 1 ), or not (class 2). This trivially introduces high classifier performance around these timepoints. However, we were not interested in the classifier's performance to differentiate in- and out-field conditions. Rather, we tested whether brain time warped data increased periodicity in classifier performance at the warping frequency. We brain time warped 2.5 seconds of data (from 1.25 seconds before to after $\mathrm{t}=0$ ). The number of trials per session ranged from 60 to 168 (mean: 104.8 trials, standard deviation: 39).

\section{Brain time warping}

The average waveshape of LFP theta was asymmetric, so we generated the clock time template signal (used to detect segments of nonstationarity in LFP theta) based on a smoothed version of the average waveshape rather than a symmetric sine wave (which is the default method). This optimizes brain time warping by reducing differences between the clock and brain time signal, improving the accuracy of the warping path (see Toolbox methods). In 3.2.3, we show TGMs and their autocorrelation map for each session. The prepared data and scripts used for analysis are included in the Brain Time Toolbox.

\subsection{Human}

We brain time warped a human dataset obtained from Bae \& Luck (2019), which is available for download at https://osf.io/bpexa/. In this study, electroencephalography (EEG) was recorded while participants viewed random dot kinematograms, where a proportion of dots moved coherently toward a direction in $360^{\circ}$ space. Each trial contained, in order, a fixation period of $1.5 \mathrm{~s}$, a motion period of $1.5 \mathrm{~s}$, and a direction report of variable duration. Again, we restrict our description to how we reformatted and analysed the data. For the full methodological details, see Bae \& Luck (2019). We hypothesized that brain time warping to occipital-parietal alpha oscillations would increase the periodicity with which a classifier is able to differentiate whether motion is leftward or rightward, resting on evidence for alpha's role in controlling spatial attention (Capotosto, Babiloni, Romani \& Corbetta (2009).

\subsubsection{Methods}

Data preparation

First, we pooled both recording sessions and coherence levels (with $25.6 \%$ and $51.2 \%$ of dots moving coherently in one direction). We used only the $1.5 \mathrm{~s}$ motion period for decoding, as we predicted the neural signature of spatial attention is most detectable during motion. We filtered each participant's data $(\mathrm{N}=16)$ to the $80 \%$ most accurate trials (defined based on the error between reported and presented motion angle), reducing the number of trials from 1280 to 1024 . This removes the subset of trials where a rare reversal effect occurred in which participants report motion in roughly the opposite direction to the presented motion. Next, we defined two classes of data based on whether motion was leftward (class 1 ; angles between $90^{\circ}$ and $270^{\circ}$ ), or rightward (class 2 ; the remaining angles).

\section{Brain time warping}

We extracted warping sources by applying ICA to the data. For each participant, we chose a component with sustained alpha power and a topographical profile that suggested an occipital-parietal origin. In 3.3.1, we show TGMs and autocorrelation maps for each participant. 


\section{Toolbox methods}

\subsection{Introduction}

The Brain Time Toolbox, abbreviated braintime, is a MATLAB software library designed for use with electroencephalography (EEG), magnetoencephalography (MEG), and single and multiunit intracranial recordings. The toolbox has two primary uses: brain time warping data structures (transforming them from clock to brain time; operation 1), and testing for periodic patterns in the data that reflect dynamic cognition (operation 2). The standard pipeline is to brain time warp data and test for a change in periodicity at frequencies of interest (Supplementary Figure 2). However, each operation can be carried out without the other - brain time warped data may be analysed outside the toolbox and periodic patterns may be tested on data that has not undergone warping (clock time data).

In this manuscript, we describe braintime in extensive technical detail with limited emphasis on user flow. For a practical guide, we recommend the more concise and step-by-step documentation on braintime's Github page (https://github.com/sandervanbree/braintime). For a quick start, we recommend braintime's tutorials.

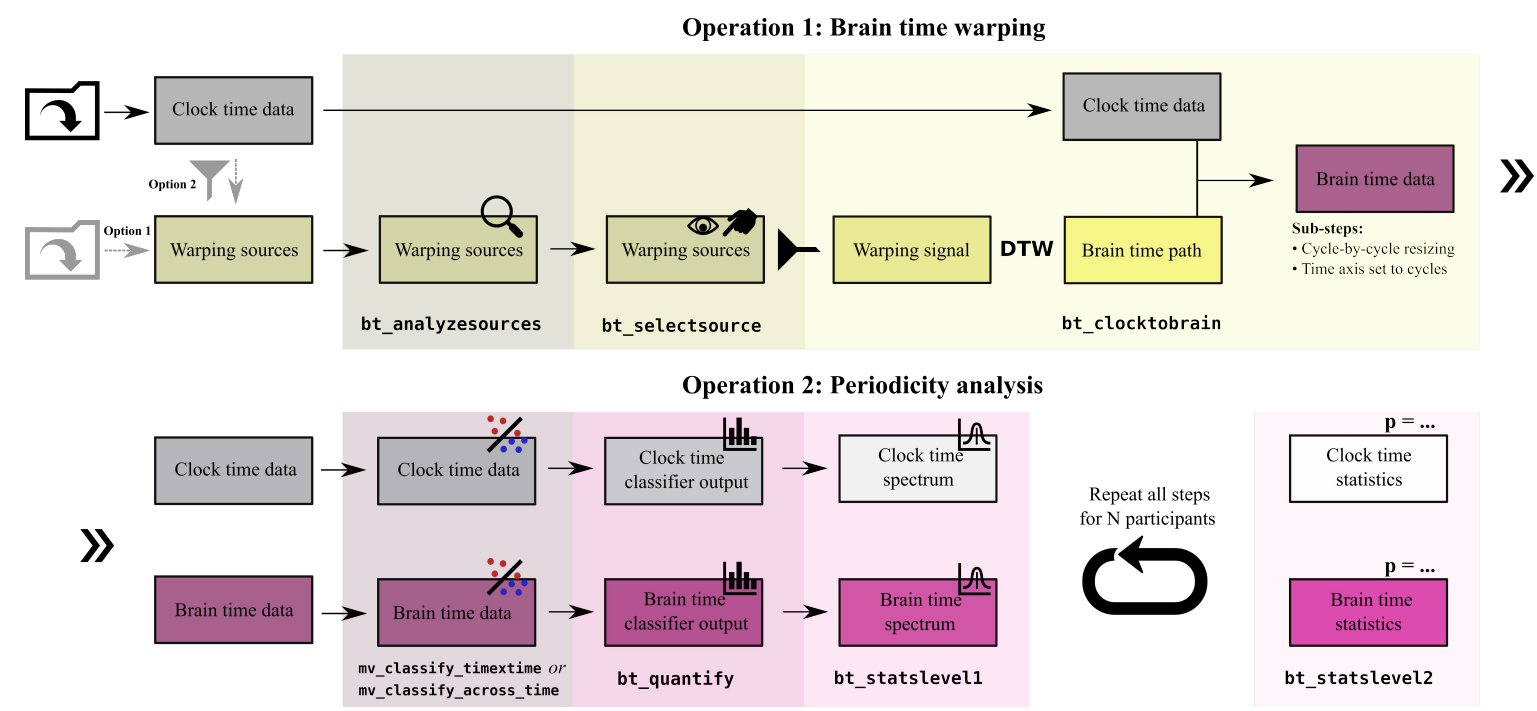

Supplementary Figure 2: the default Brain Time Toolbox pipeline

Braintime has two primary operations. (Top row) For brain time warping, the user first loads a FieldTrip formatted data structure that will be transformed (clock time data). Separately, a data structure with warping sources is required. This structure contains potential warping signals - the oscillation used to transform the data. The warping sources may be loaded separately (option 1) or extracted from the clock time data (option 2). bt_analyzesources analyzes each warping source for warping signals based on user parameters. Next, using bt_selectsource, the user can inspect each warping source's characteristics (such as spectral information) and designate a warping signal as brain time. Finally, bt_clocktobrain dynamically time warps (DTW) the warping signal to either a stationary sine wave or the warping source's average waveshape. The resulting warping path is applied to the clock time data, cycle-bycycle, and resized to the original length. The function also sets the time axis from seconds to cycles of brain time. (Bottom row) For the periodicity analysis, braintime first calls MVPA Light to test a classifier on two classes of data, prespecified by the user. The resulting output is one dimensional for mv_classify_across_time, or two dimensional, when testing for time generalization of the classifier using mv_classify_timextime. Then, across this output, bt_quantify tests for periodic patterns in classification performance, resulting in a periodicity spectrum. Next, bt_stats level1 performs first-level statistics by comparing the empirical spectrum with a user-specified number of permuted spectra (obtained by shuffling class labels). Finally, bt_statslevel2 obtains group level results of the classifier's periodicity, and calls MVPA Light to perform cluster correction over the classifier's performance.

\subsubsection{Requirements}

Braintime interfaces with the following software packages, requiring each to be installed:

- MATLAB Signal Processing Toolbox (The MathWorks)

- FieldTrip (Oostenveld et al., 2011)

- MVPA Light (Treder, 2020) 
Besides these software dependencies, braintime requires the electrophysiology data to be structured in a FieldTrip format. For data structures that are not in this format, FieldTrip has built-in tools to convert a wide array of common file types. See the FieldTrip documentation for information on how to convert data.

In case braintime's second operation will be used (testing for periodicity), the input clock time data needs to consist of two classes of data hypothesized to yield the neural signature of the studied cognitive process. Moreover, trials in the data structure need to be labelled based on their class membership. For more details on how to do this, see section 2.3.

\subsubsection{Download and installation}

Before setting up braintime, ensure FieldTrip and MVPA Light are downloaded and fully initialized (download links above). Braintime can be downloaded on the toolbox Github page. First, download the ZIP folder and unzip it to a preferred path. Then, run the function setup_braintime (in the setup folder). To get started with the toolbox, see braintime's tutorials.

\subsection{Operation 1: Brain time warping}

Brain time warping transforms data according to the phase dynamics of an oscillation hypothesized to clock the studied cognitive process. This changes the time axis of the data from seconds to cycles of the warping frequency.

\subsubsection{Obtaining warping sources}

Warping sources may either be ad hoc extracted from the clock time data or loaded from a separate data structure. The warping sources contain warping signals - potential oscillations used for brain time warping. There are only two restrictions to what constitutes warping sources: the data structure needs to be in a FieldTrip format, and the data needs to align in time with the to-be-warped clock time data. Users are encouraged to decide on a case-bycase basis what data serve as the best warping sources. Below, we describe two methods of obtaining warping sources, and provide several examples for each.

\section{Ad hoc extraction}

Warping sources may be extracted from clock time data on the fly, for example using independent component analysis (ICA). ICA is a popular method to decompose electrophysiology data into additive subcomponents (Makeig, Bell, Jung \& Sejnowski, 1995). The resulting components aggregate data with statistically uncorrelated spectral characteristics, making for suitable warping sources. Each component contains potential warping signals. The toolbox automatically detects when the warping sources are ICA components, and suggests removal of the selected component from the brain time warped data when continuing analyses outside the toolbox. For singleand multiunit recordings, an example of ad hoc extraction is to separate one (or more) local field potential channels into a warping source(s) structure.

\section{Preloaded data structure}

Warping sources may also be obtained from a separate preloaded data structure, in the form of previously extracted data. An example could be a study where magnetometer and gradiometer channels underwent separate preprocessing steps, with their data saved in separate files. Or perhaps a user has created a set of virtual channels using source localization, intending to warp sensor-level data using source-level phase estimation. Warping sources may also be obtained from a separate recording altogether. For example, a user may opt to warp EEG data using warping signal obtained from MEG data, or vice versa.

\subsubsection{Analysing warping source}

In bt_analyzesources, braintime uses FieldTrip to perform time frequency analyses on each warping source. Users may choose between a variety of time frequency analysis parameters based on FieldTrip's ft freqanalysis, and may opt to include $1 / \mathrm{F}$ correction. In addition, users select a range of frequencies predicted to contain the coordinating oscillation. For example, a user interested in spatial attention may specify a range of 8 to $12 \mathrm{Hertz}(\mathrm{Hz})$. The toolbox estimates the phase of high power oscillations in this range in anticipation of brain time warping. Upon completion of the time frequency analyses, the toolbox sorts the warping sources based on power in the range of interest. Optionally, for EEG and MEG, the sorting of warping sources may be weighted by the hypothesized topographical activity of warping sources. For example, a researcher may want to focus on warping sources with parietal activity, or a lateralized source. To enable topography weighting, users first call bt_templatetopo and draw a topographical profile on a template layout. Braintime then loads the 
template topography and adapts the ranking of warping sources to their topography's correlation with the template topography, instead of the default setting which sorts solely by maximum power at frequencies of interest.

\section{Asymmetry and waveshape}

For each warping source, braintime detects the average waveshape and the asymmetry of each cycle in it. This allows users who have predictions about the symmetry of their coordinating oscillation to decide between warping sources. In addition, the amount of asymmetry may also inform parameter selection during subsequent steps in braintime.

The waveshape and asymmetry of data are obtained via the following steps. First, braintime applies a band-pass filter in a 2 Hertz $(\mathrm{Hz})$ window around the warping frequency. Then, trial-by-trial, the toolbox finds local minima and maxima in the filtered data, reflecting troughs and peaks, respectively. Then, based on the location of these minima and maxima, braintime extracts associated data in the (non-filtered) warping sources, calculating a peak-triggered wave. Thus, the peaks are located in the filtered data, while the shape of waves themselves are obtained by extracting data samples in the raw data around the peak locations. This is repeated for all cycles. Then, the asymmetry is calculated for each cycle, while the average waveshape is obtained by averaging each cycles' data. We defined asymmetry as the difference in duration between the ascending and descending flank of a cycle, normalized by its duration (Belluscio et al., 2012):

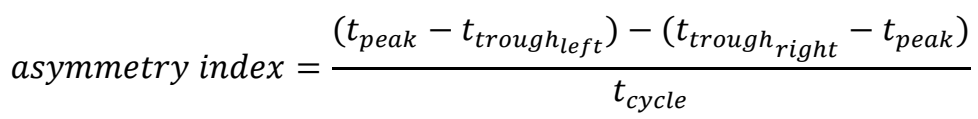

\subsubsection{Selecting a warping signal}

In bt_selectsource, the user selects a warping source and its associated warping signal, designating brain time. In this step, braintime creates a graphical user interface that displays each warping source's information as calculated by bt_analyzesources (Supplementary Figure 3). This information comprises (1) a textbox with recommended criteria for warping source selection and browsing instructions, (2) the topography of the warping source (if requested), (3) the asymmetry and shape of the average waveshape, (4) the time frequency representation of the warping source's data, and (5) its power spectrum. Users may visually inspect each warping source and combine all information to designate the warping signal predicted to clock the studied cognitive process of interest, in keeping with a hypothesis-driven approach.

\subsubsection{Warping clock to brain time}

In bt_clocktobrain, the phase of the selected warping signal is used to warp the clock time data structure (Supplementary Figure 4). Before describing how dynamic time warping (DTW) is employed, we first explain two parameters that determine what DTW is applied to.

\section{Warp method}

We define clock time as a stationary oscillation, oscillating away faithfully to seconds. This is what the warping signal will be dynamically time warped onto. We implement two ways of generating this stationary oscillation. First, users may choose the default method, where a stationary sine wave is generated with MATLAB. However, for data with asymmetric waveshapes, using a sine wave with perfect symmetry may impede DTW's attempt to minimize Euclidean distance between the phase of clock and brain time. Thus, we include the option to warp using a concatenation of the smoothed average waveshape of the warping source, yielding a sinusoid with matching asymmetry (Supplementary Figure 4).

\section{Phase method}

Besides control over what constitutes clock time phase, users may opt to estimate brain time's phase in two ways. The standard way of estimating brain time's phase is using Fast Fourier Transform (FFT) of band-pass filtered data around the warping frequency in the selected warping source. However, the toolbox also offers Generalized Eigenvalue Decomposition (GED) as an alternative method to estimate the warping frequency's phase holistically across all warping sources. This method is appropriate when the warping sources are all considered to contain information about brain time. For example, the user's warping sources may be local field potential channels in the hippocampus. In this case, the user may not want to choose one local field potential channel given their interest in the phase of theta oscillations across the entire hippocampus. Similarly, a user may have source localized parietal cortex channels which each provide valuable phase information. In these cases, GED is the optimal choice. 
bioRxiv preprint doi: https://doi.org/10.1101/2021.06.09.447763; this version posted August 12, 2021. The copyright holder for this preprint (which was not certified by peer review) is the author/funder, who has granted bioRxiv a license to display the preprint in perpetuity. It is made available under aCC-BY-ND 4.0 International license.

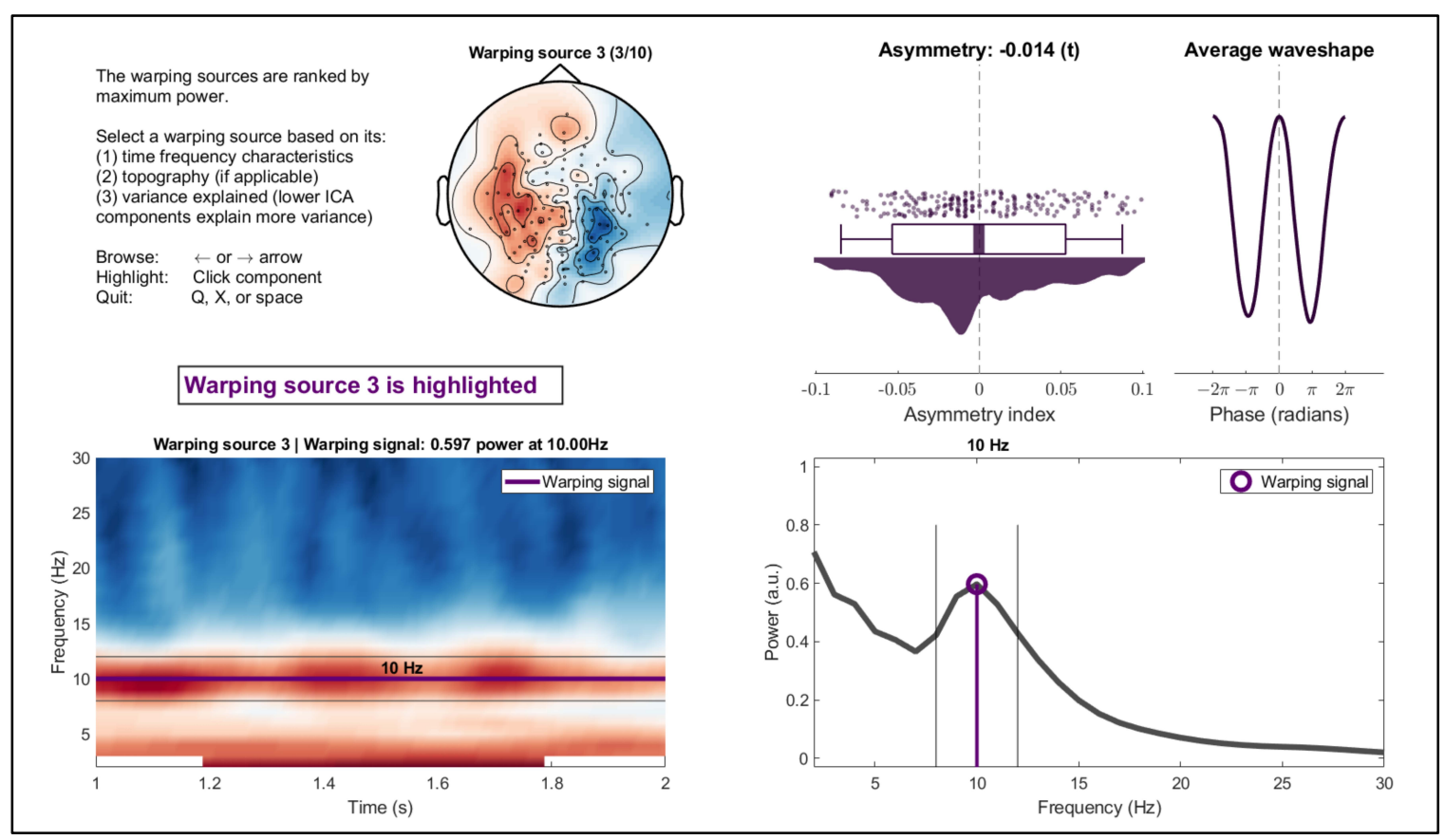

Supplementary Figure 3: Selecting a warping source

The graphical user interface generated by bt selectsource. (Top row) A textbox is displayed with suggested criteria for warping source selection, as well as instructions for browsing. Next, optionally for EEG and MEG, the topography is displayed. On the right, the average asymmetry of cycles in each warping source is displayed (one dot represents one cycle in the warping source). This serves two purposes: users may want to discriminate between sources based on their asymmetry, or check levels of asymmetry to inform parameter selection during subsequent steps. (Bottom row) The warping source's time frequency representation is displayed, along with the power spectrum. For both plots, the borders of the frequency range of interest and the location of the warping signal are highlighted.

GED finds a spatiotemporal filter that differentiates all the warping source data into components with high and low power of the warping frequency (Parra, Spence, Gerson \& Sajda, 2005; Cohen, 2017). When users specify this option, braintime extracts the component with the highest weight, yielding a time series of the GED component. The toolbox takes the angle of its Hilbert transform, defining brain time. To perform GED, the toolbox uses a modified version of the functions provided by Mike X Cohen (reported in Cohen, 2017). In short, FFT offers a classical and source-specific phase estimation of the warping frequency, while GED estimates the warping frequency's phase holistically across warping sources.

\section{Brain time warping}

With both the clock time and brain time phase defined and computed, braintime initiates brain time warping. Here, the toolbox dynamically time warps clock and brain time onto each other. Clock time, here, is the unwrapped phase of a stationary sinusoid or average waveshape, while brain time is the unwrapped phase of the warping signal (estimated within a source using FFT or across sources using GED). These two phase vectors are dynamically time warped using MATLAB's dtw command. DTW (Sakoe \& Chiba, 1978) computes the optimal alignment between two signals, minimizing their Euclidean alignment. For a formal specification of DTW, see Berndt \& Clifford, 1994 and Sakoe \& Chiba, 1978.

The toolbox extracts the warping path between clock time (ct_phs) and brain time (bt_phs) using the following MATLAB code:

$$
[\sim, i x, i y]=d t w\left(b t \_p h s, c t \_p h s\right)
$$

ix is the warping path which, when applied to bt_phs, will minimize its distance to ct_phs, and vice versa for iy. Since ct_phs is a stationary signal, ix indicates (1) segments of nonstationarity in bt_phs (2) and how bt_phs needs to be resampled to become a stationary signal. Crucially, rather than applying ix to bt phs to get it closer to ct_phs, brain time warping instead applies ix to the clock time data structure, transforming all of its dynamics based on the nonstationarities in the warping signal. The result is a data structure with dynamics 
bioRxiv preprint doi: https://doi.org/10.1101/2021.06.09.447763; this version posted August 12, 2021. The copyright holder for this preprint (which was not certified by peer review) is the author/funder, who has granted bioRxiv a license to display the preprint in perpetuity. It is made available under aCC-BY-ND 4.0 International license.

adjusted in accordance with the temporal evolution of oscillations predicted to coordinate cognitive function, enabling an improved readout of its dynamics.

Whilst applying each trial's ix to transform clock time data, the toolbox implements a cycle-by-cycle resizing. This is to ensure that the data of each cycle per trial has a constant number of samples. Without this step, if the warping path contains an extended segment of data repetitions for some trials, this would stretch out the data extensively, introducing a misalignment of brain time across trials. In other words, cycle-by-cycle resizing ensures brain time unfolds with a similar timecourse across trials by aligning its cycles. To resize data, the toolbox uses MATLAB's imresize (which uses nearest-neighbour interpolation). Braintime determines the start and end of cycles using the warping path iy (the warping path from ct phs to bt_phs), which has an equal length to ix but more closely reflects the stationary dynamics of clock time. An additional side-effect of cycleby-cycle resizing is that the number of samples of the original data remains unchanged after brain time warping.

Upon completion of these steps, the data is in brain time. In light of this, the toolbox changes the time axis from milli(seconds) to cycles of the warping signal. Next, users may test for dynamic information patterns using operation 2 of the toolbox or continue analysis outside of the toolbox. In case of the latter, there are some circularity concerns that will be addressed in section 2.4.1. Before that, we describe the toolbox' second operation.

A.
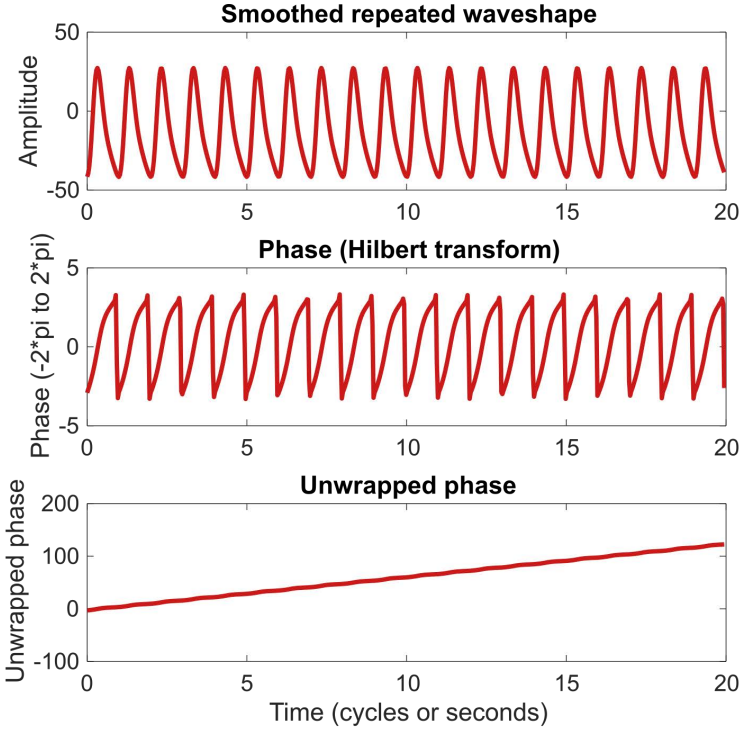

Supplementary Figure 4: Warping clock to brain time Braintime prints a series of plots upon completion of bt_clocktobrain to show the application of brain time warping to the data at hand. (A) In this example, the user has set braintime to warp to the smoothed average waveshape of the selected warping source (top). The toolbox extracts the angle of its Hilbert transform (middle) and unwraps the phase in preparation for warping (bottom). (B) The unwrapped phase vector of the warping signal and the stationary sine wave (representing clock time) are displayed (top), and so is their alignment after applying dynamic time warping (bottom). (C) Here, the warping path has been applied to the original clock time data, yielding a dynamic resampled version of the original data based on the brain's dynamics.
B.
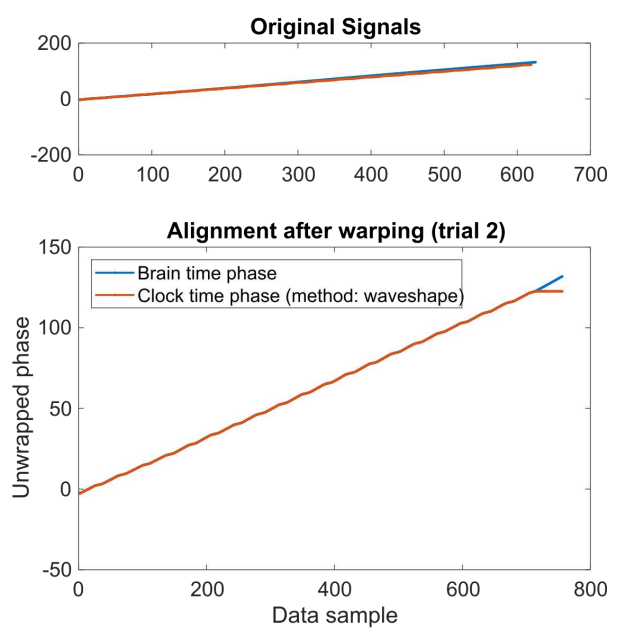

C.

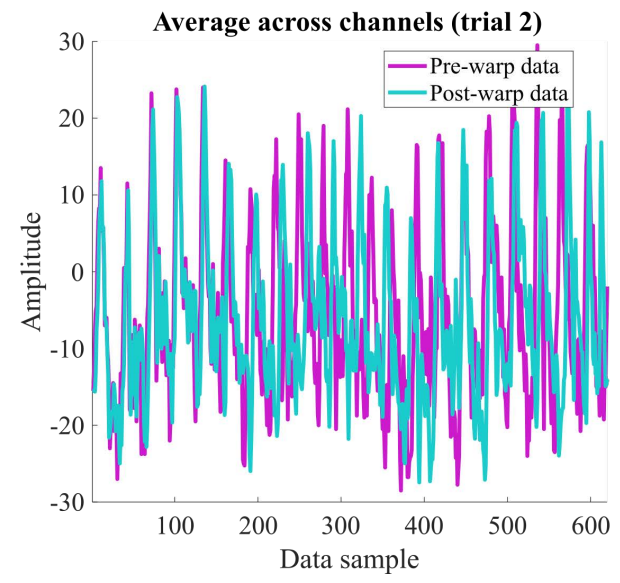

\subsection{Operation 2: Periodicity analysis}

Braintime allows users to test for dynamic patterns of information using machine learning techniques. This requires the data to contain two classes of conditions, which, when taking their difference in neural activity, reflects the studied cognitive process. We define this difference as the neural signature. The assumption of operation 2 is this: if the warping signal clocks the cognitive process, then the warped data should show an increase in the periodic dynamics of the neural signature. Thus, we compare the periodicity of a classifier's performance 
between clock and brain time data to test for a difference in such patterns. The general pipeline is that each participant's clock and brain time data undergoes classification (2.3.1), quantification of classifier performance (2.3.2), and first-level statistics (2.3.3). Then, second-level statistics can be initiated on the first-level results (2.3.4).

\section{Requirements}

To get started with the second operation, both the clock time and brain time warped data need to have each trial's class membership labelled. This is necessary for MVPA Light and braintime to understand the class structure present in the data. Braintime's tutorials describe how to do this using example code, but briefly, the user sets bt_data.trialinfo and ct_data.trialinfo to a vector of 1's and 2's in line with the trial structure (class labels). For example, for a dataset on motor processing, a triplet of trials that are in left, right, and left movement conditions would need to be labelled as [ll $\left.\begin{array}{lll}1 & 2 & 1\end{array}\right]$ or [ [ $\left.\begin{array}{lll}2 & 1 & 2\end{array}\right]$.

When using the second operation, it is important for users to establish the absence of high-pass filtering artefacts in the data. In a recent publication, it is reported that high-pass filtering may result in temporal displacement of information, causing leakage of classification performance (van Driel, Olivers \& Fahrenfort, 2021). These artefacts may influence the periodicity analysis. In light of this, we suggest avoiding high-pass filtering when the data is clean. Alternatively, trial-masked robust detrending may be applied as an artefact-free method of removing low frequencies (van Driel et al., 2021). Finally, if users do require high-pass filtering, we recommend a careful comparison between classification performance in the filtered data and non-filtered data to establish whether artefacts are present. Users are made aware of potential high-pass filtering artefacts in all sources of braintime's documentation.

\subsubsection{Classifying the data}

To perform pattern classification, braintime calls MVPA Light (Treder, 2020). MVPA Light can be used to perform multivariate pattern analysis using different models (e.g., support vector machines, logistic regression, and linear discriminant analysis; LDA), classification metrics (e.g., accuracy, area under the curve, fidelity values), and cross-validation methods (e.g., k-fold, hold-out, leave one out). Classification may be performed in the standard way using mv_classify_across_time or with temporal generalization using mv_classify_timextime (Supplementary Figure 5). The former results in a one-dimensional (1D) time series that reflects how a classifier trained on each timepoint performs when tested on that timepoint in held out data. The latter results in a two-dimensional (2D) temporal generalization matrix (TGM) that reflects how performance generalizes from each trained timepoint to all other timepoints in held out data (King \& Dehaene, 2014). The diagonal of the TGM is identical to $1 \mathrm{D}$ classification, while the off-diagonal provides information about generalization of performance. This provides information about the degree of constancy of the neural code ("temporal generalization"). As such, mv_classify_across_time is appropriate when the neural signature is predicted to evolve beyond classifier recognition from one moment to the next, while mv_classify_timextime is appropriate when there is some constancy of the neural code across time. For analysis with braintime, if some constancy is expected, we strongly recommend mv_classify_timextime as it yields exponentially more data to perform periodicity analysis over - substantially increasing the power of the analysis.

\subsubsection{Quantifying classifier periodicity}

In bt_quantify, classifier periodicity is quantified by an FFT over the classifier timecourse (1D results), or over each row and column of the time generalized classifier performance (TGM; 2D results). We use FieldTrip to apply the FFT using a multitaper frequency transformation (using discrete prolate spheroidal sequences and Hanning window tapers). The FFT is performed across frequency ranges specified by the user.

For the time generalized classifier performance, users can specify whether the periodicity is done over the rows and columns of the TGM itself, or over its autocorrelation map (Supplementary Figure 5). The autocorrelation map correlates the TGM with all shifted versions of itself. That is, it iteratively shifts the TGM in each $\mathrm{X}$ and $\mathrm{Y}$ direction and plots the correlation of the shifted map with itself in coordinate space. The most powerful option depends on the uniformity of periodic patterns. Generally, the autocorrelation map is a more powerful approach, as it accentuates periodic patterns that are present in the TGM. However, when multiple periodicity rates are present in the TGM, the autocorrelation may drown out the weaker periodicity. Similarly, when periodicity is present in only a small part of the TGM, changing its rate or disappearing across time, the autocorrelation map may fail to detect it. In short, when uniform periodicity patterns in the data are expected, 
bioRxiv preprint doi: https://doi.org/10.1101/2021.06.09.447763; this version posted August 12, 2021. The copyright holder for this preprint (which was not certified by peer review) is the author/funder, who has granted bioRxiv a license to display the preprint in perpetuity. It is made available under aCC-BY-ND 4.0 International license.

analysing the autocorrelation map of TGMs is a powerful approach. When the pattern is expected to only be present partially, or when multiple rates are predicted, it is more powerful to analyse the TGM itself.
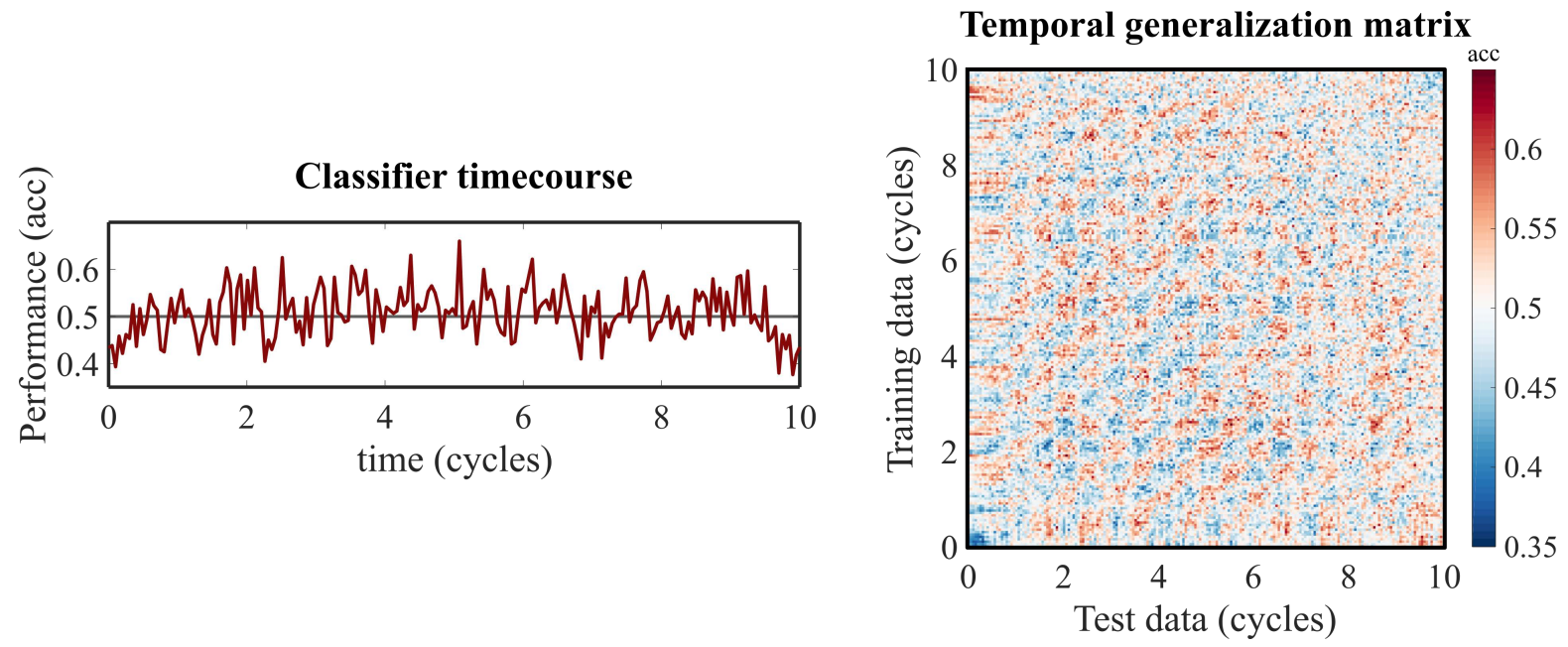

Supplementary Figure 5: Two types of classification

Braintime calls MVPA Light to perform standard classification across time (1D; left) or temporal generalization (2D; right). The classification timecourse on the left equals the diagonal of the TGM on the right. The off-diagonal represents how well classification generalizes when trained on one timepoint and generalized to each other timepoint in held-out data. Braintime can perform periodicity analyses over both types of classification. (acc $=$ accuracy).
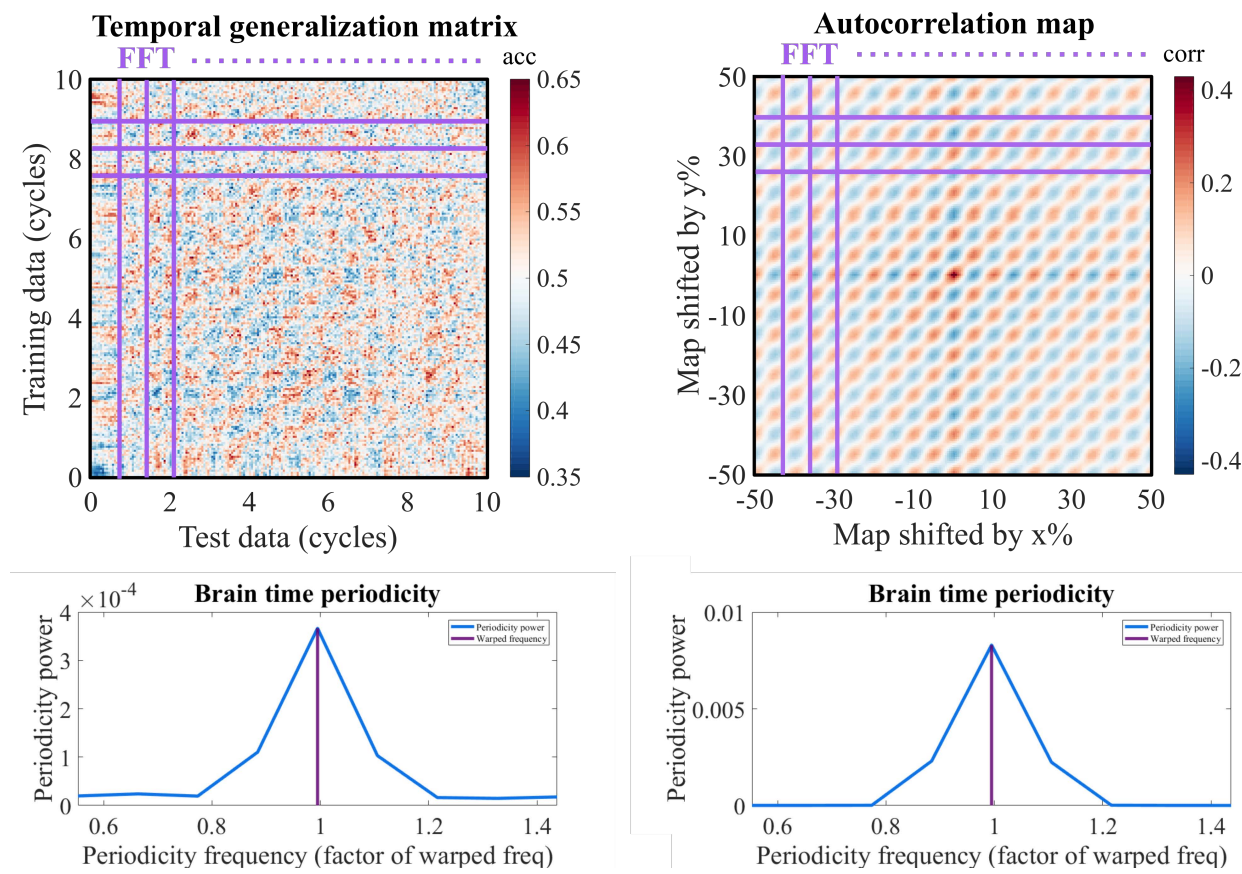

Supplementary Figure 6: Two ways of performing 2D periodicity analysis

(Top) Row-by-row and column-by-column, braintime performs an FFT over the TGM by itself, or its autocorrelation map. The former is more powerful when periodicity is present in small segments of the TGM, or if multiple periodicity frequencies are present. The latter is more powerful when the periodic pattern is present in most of the TGM (as is the case in this dataset). (Bottom) The resulting power spectra are averaged across all rows and columns, resulting in one participant's "periodicity spectrum". (corr = correlation) 


\section{Periodicity referenced to brain time}

For brain time data, just as the time axis has been changed from seconds to cycles, periodicity is redefined from clock time to brain time. Specifically, each participant's periodicity is normalized to their warping frequency $\left(\frac{\text { default frequency }}{\text { warping frequency }}\right)$. For example, if a user decides to quantify periodicity between 5 and $13 \mathrm{~Hz}$ for a participant with a $9 \mathrm{~Hz}$ warping frequency, the resulting brain time periodicity will range from between $0.56 \mathrm{~Hz}\left(=\frac{5 \mathrm{~Hz}}{9 \mathrm{~Hz}}\right)$ and $1.44 \mathrm{~Hz}\left(=\frac{13 \mathrm{~Hz}}{9 \mathrm{~Hz}}\right)$, see Supplementary Figure 6 (bottom). This referencing to brain time allows participants to be compared with reference to each brain's own dynamics. For example, if periodicity across participants were to cluster at $0.5 \mathrm{~Hz}$, this would mean participants tend to show periodic patterns in their neural signature at half the rate of their clocking oscillations.

\subsubsection{First-level statistics of periodicity}

In bt_statslevel1, quantified periodicity is subjected to first-level statistics to establish its statistical robustness. The user specifies a range of frequencies that will be tested, and the desired number of first-level permutations (numperms1). Braintime obtains a null distribution by repeating data classification (2.3.1) and quantification of its periodicity (2.3.2) numperms 1 times with the class labels shuffled randomly (destroying correspondence between trials and their associated condition). This yields a pool of permuted periodicity spectra that offers a confidence interval for each frequency. P-values are not provided until the second-level statistics are completed.

\subsubsection{Second-level statistics of periodicity}

Upon completion of the previous steps for all participants, users are requested to combine the first-level periodicity data structure of each participant into one superstructure. Next, in anticipation of bt_statslevel2, users specify the number of second-level permutations (numperms2) and the desired method of multiple testing correction across periodicity frequencies (false discovery rate; FDR, Bonferroni correction, or no correction). bt_statslevel2 undertakes the following steps:

\section{Z-scoring}

Braintime performs z-scoring on each participant's periodicity spectra for two reasons. First, the output of FFT has amplitudes with arbitrary values, creating arbitrary differences in amplitude between participants. Second, we want to avoid participants with high periodicity (even after correcting for arbitrary differences) to drive a group effect. The toolbox implements z-scoring by normalizing both the permuted spectra and empirical spectra to the mean and standard deviation of the permuted spectra (i.e., by subtracting the mean and dividing by the standard deviation). With this method, the permuted spectra are considered a noise floor for which the empirical spectra are corrected.

\section{Filtering to common frequencies}

As discussed before (2.3.2), participants' range of tested periodicity frequencies are normalized to their warping frequency. This creates some variability in the range of tested frequencies across participants. For second-level statistics, braintime filters each participant's spectra to the frequencies that are common to all participants. To overcome potential variations in the frequency resolution between participants, braintime uses imresize to interpolate all participants' spectra according to the mode spectral resolution across participants.

\section{Obtaining p-values}

On the second-level, each clock and brain time periodicity frequency is statistically tested across participants. This is done in the following way. For each participant, the toolbox grabs one random spectrum of each participant's numperms 1 permuted spectra. This is repeated numperms 2 times, yielding a pool of numperms 2 new spectra. For each frequency, braintime compares the average empirical periodicity power to the distribution of power in the numperms 2 pool spectra. The p-value of each frequency is defined as the proportion of numperms 2 power values that are equal or higher to the average empirical power value $\left(\mathrm{p}=\frac{N}{\text { numperms } 2}\right.$, where $\mathrm{N}$ is the number of times the permuted pool equals or exceeds empirical power). 
Users may correct for multiple comparisons (where the comparisons are the tested frequencies) by using false discovery rate (FDR) or Bonferroni correction. For brain time spectra, three frequencies are exempted by default for multiple testing correction because, in line with the hypothesis-driven approach of braintime, periodicity is a priori expected at these rates. Specifically, these are at $0.5 \times, 1 \times$, and $2 \times$ the warping frequency (i.e., $0.5 \mathrm{~Hz}, 1 \mathrm{~Hz}$, and $2 \mathrm{~Hz}$ ) in the brain time spectra. Depending on both the underlying dynamics and the classifier method used (mv_classify_across_time ormv_classify_timextime), periodicity is predicted at one or more of these rates (if the warping signal clocks the cognitive process of interest). In this sense, braintime performs two statistical tests: one primary test across the three hypothesized frequencies, and an additional one across the specified frequency range of interest. In the second-level periodicity spectra, the uncorrected p-values at the three frequencies of interest are combined with the corrected p-values of the remaining frequencies of interest.

\section{Cluster statistics}

Besides establishing the statistical robustness of periodicity in the classifier's performance, braintime also displays robustness of classifier performance per se. That is, it displays at which timepoints the classifier is able to differentiate the two classes of data above chance. The toolbox calls MVPA Light to perform cluster correction over the results obtained from mv_classify_across_time or mv_classify_timextime. Users may specify a variety of parameters for cluster correction that are read and implemented by MVPA Light, including test methods (e.g., binomial or permutation based-cluster correction) as well as the number of permutations used to determine significant clusters. MVPA Light implements cluster correction as described by Maris \& Oostenveld (2007). More information on cluster correction in MVPA Light can be found on its Github page.

\subsection{Methodological considerations}

Here, we describe several methodological concerns and considerations when using braintime.

\subsubsection{Circularity concerns}

As mentioned in the main text, a concern with brain time warping could be that it trivially imposes oscillatory structure at the warping frequency, causing the neural signature of a cognitive process to fluctuate even when warping to oscillations that do not clock it. A first point is that the warping path generated by DTW is not oscillatory, but instead represents how the warping signal needs to be transformed to reduce its nonstationarity. That is to say, application of the warping path to a random signal is not expected to alter its oscillatory structure in any way. Nevertheless, the concern persists. If the warping signal is present in the data, the oscillatory structure of the data is likely enhanced because the warping signal has its nonstationarities removed as a consequence of the path being applied to all the data. Below, we describe for which analyses circularity could be a concern, and how it can be avoided.

\section{Analysis}

Circularity is not a concern when testing for periodic patterns using the toolbox (operation 2). This is because of braintime's method of obtaining a null distribution against which empirical periodicity is tested. Specifically, the only difference between this permuted pool and the empirical data is the shuffling of class labels. Besides this difference in data-to-condition allocation, the trials themselves remain unchanged. As a consequence, any trivially imposed oscillatory structure into the data is present equally between the permuted and empirical set. To the extent a classifier is able to exploit circular patterns (if they are present), it will be able to do so for both dimensions. Thus, where circularity is present, it is expected to cancel out through braintime's statistical method.

However, when using brain time warped data outside the toolbox, it may be that a researcher's analyses tap into the circular patterns (if they are present). Following the same logic, circularity is an issue to the extent it differentially affects the empirical and null distribution.

\section{Dependence}

Since circularity may arise from the warping signal, whether circularity is present at all critically depends on whether the warping signal is present in the transformed data. We call this concept data dependence. If the warping signal is present in the transformed data, the transformed data are said to be dependent. Below, we provide several examples with varying levels of dependence. Most caution and vigilance is needed for highly dependent data. To emphasize, the level of dependence is only relevant when analysing brain time warped data outside braintime, and only with analyses that can exploit circular patterns. 
bioRxiv preprint doi: https://doi.org/10.1101/2021.06.09.447763; this version posted August 12, 2021. The copyright holder for this preprint (which was not certified by peer review) is the author/funder, who has granted bioRxiv a license to display the preprint in perpetuity. It is made available under aCC-BY-ND 4.0 International license.

\section{High dependence}

H1) The warping signal was obtained from a channel in the clock time data, and it is still present in the brain time data.

H2) The warping signal was obtained from an ICA component in the clock time data, and it is still present in the brain time data.

\section{Medium dependence}

M1) The warping signal was obtained from source localized virtual channels obtained from within the clock time data, and these channels are still present in the brain time data.

M2) The warping signal comes from a set of anterior channels of an EEG or MEG dataset, which was used to warp posterior sensors.

M3) The warping signal is from high pass filtered MEG data, which is used to warp low pass filtered data.

\section{Independence}

These include all cases where the signal used for warping is absent from the brain time data. For example: L1) The warping signal is obtained from local field potentials (LFP) in multi-unit recordings, which is used to warp single-cell recordings.

L2) The warping signal is from an ICA component that is absent in the brain time data.

L3) The warping signal is from a channel that is absent and spectrally orthogonal to channels in brain time data.

\section{Solution}

Circularity can be avoided by removing the warping source from the brain time warped data. An obvious example is the case where the warping source structure comprises ICA components (H2). Braintime automatically detects when warping sources are ICA component, and both recommends their removal and assists in this process $(\mathrm{H} 2 \rightarrow$ L2). Similarly, most of the aforementioned examples can be made independent. For example, in example M1, the spatial filters used for virtual channel generation could be used to filter those channels from brain time warped data. Or, if a brain time warped data structure comprises both LFP and single-unit channels, and the LFP channels were used to warp, then these channels can be removed from the brain time warped data.

\subsubsection{Warping artefacts}

Sometimes, brain time warping introduces a long series of sample repetitions at the start and/or end of the data that are too extended for the cycle-by-cycle resizing operation to compress them (Supplementary Figure 7). These extended data repetitions tend to occur at the start of brain time warped data, because this is where DTW attempts to repair initial differences in the phase between clock time and the warping signal. In other words, typically clock and brain time start out of tune, and so brain time warping repeats brain time data from the get-go until the two dimensions fall back in tune. These data repetitions are not intrinsically artefactual - they represent a true mismatch between the brain's dynamics and clock time that brain time warping accounts for. Nevertheless, they could in principle affect subsequent analyses, such as braintime's periodicity analysis, causing them to exert an artefactual low-frequency footprint on it. For simplicity, we therefore refer to these data repetitions as warping artefacts.

Generally, warping artefacts are too small to exert an effect on braintime's periodicity analysis. Their effects on analyses outside the toolbox depends on the degree to which the data repetitions affect it. The toolbox has built-in tools to avoid the artefact, if so desired. Specifically, the toolbox has a "cutartefact" parameter that brain time warps an additional 0.5 seconds before and after the specified window of interest, which is subsequently removed at the end of bt_clocktobrain. Here, the data repetitions at the start (end) of the brain time warped data are shifted to the 0.5 seconds before (after) the window of interest. The advantage of this method is that the warping artefact is cut. The downside of this method is that there is a slight mismatch across trials in the true and specified time window of interest in the order of several milliseconds (for this reason, the default method that implements no artefact cutting is called "consistenttime"). Researchers may decide on a case-by-case basis which method is appropriate - cutartefact or consisttenttime - depending on the relative importance of the absence of warping artefacts and the constancy of the data's timing.

The two methods warp over different time periods and may therefore vary in their determination of which data belong to which cycle (the cycle-by-cycle resizing step, see 2.2.4). Braintime has a special function (bt_checkallocation) that visualizes how each of the two methods allocates data to cycles, allowing users to make informed decisions about which method to use and how much they diverge. 
bioRxiv preprint doi: https://doi.org/10.1101/2021 06 09.447763; this version posted August 12, 2021. The copyright holder for this preprint (which was not certified by peer review) is the author/funder, who has granted bioRxiv a license to display the preprint in perpetuity. It is made available under aCC-BY-ND 4.0 International license.

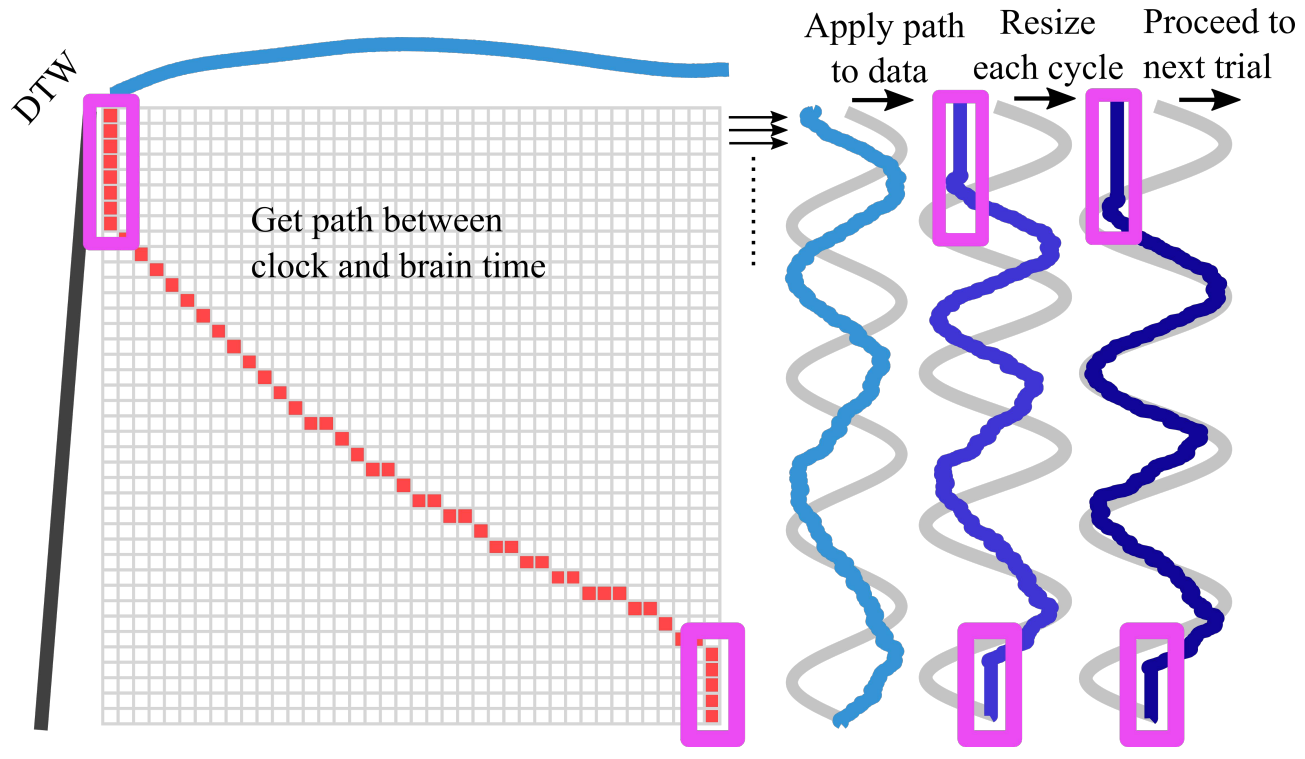

Supplementary Figure 7: Warping artefacts

Brain time warping may repeat samples for an extended period at the start and end of the data, depending the disharmony between clock and brain time at the onset of trials (purple boxes). Braintime includes a metl to remove the artefact, which warps additional data before and after the window of interest which subsequently removed. 


\section{Supplementary data plots}

For the plots of each dataset analysis, the y-axis of periodicity spectra and the colour scale of TGMs and autocorrelation maps are fixed to a constant range across (1) participants and (2) clock and brain time results to enable a visual comparison of magnitude. For a description of each type of classification plot, see section 2.3.

\subsection{Simulation}

\subsubsection{Classification (main analysis)}

Brain time TGM
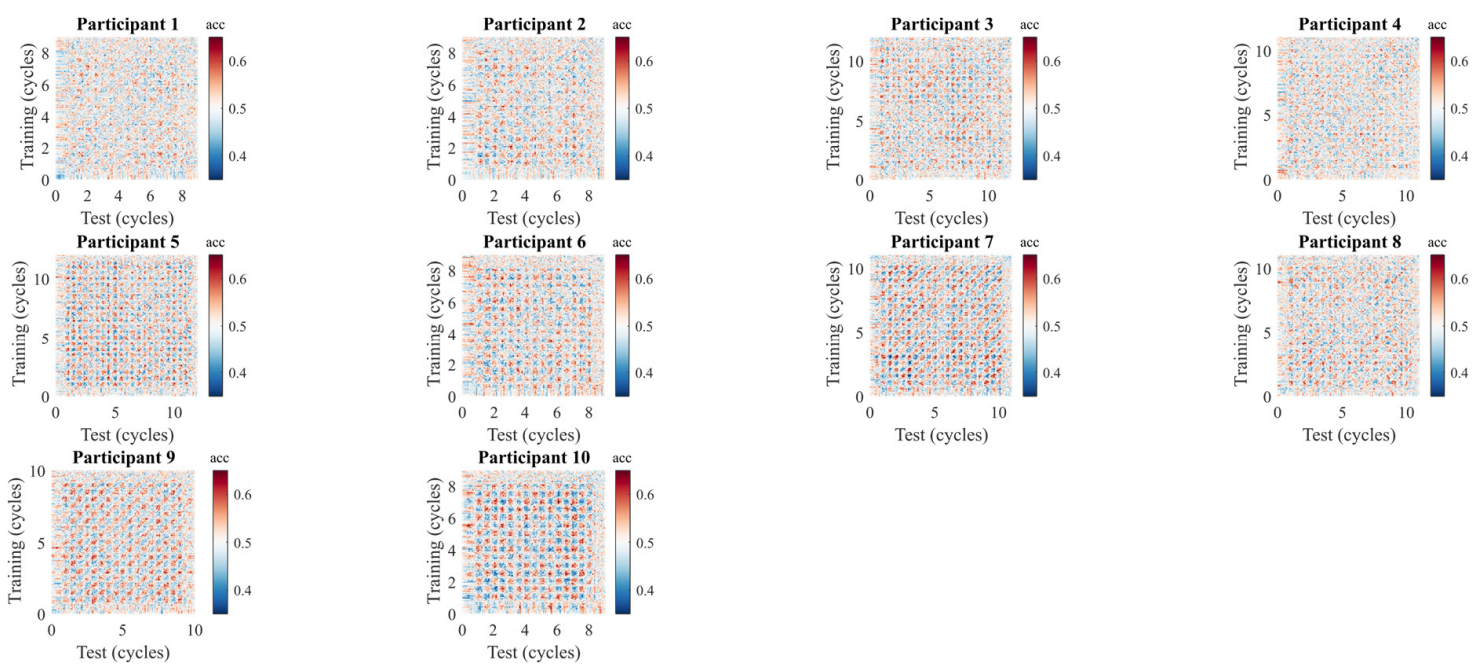

Brain time autocorrelation
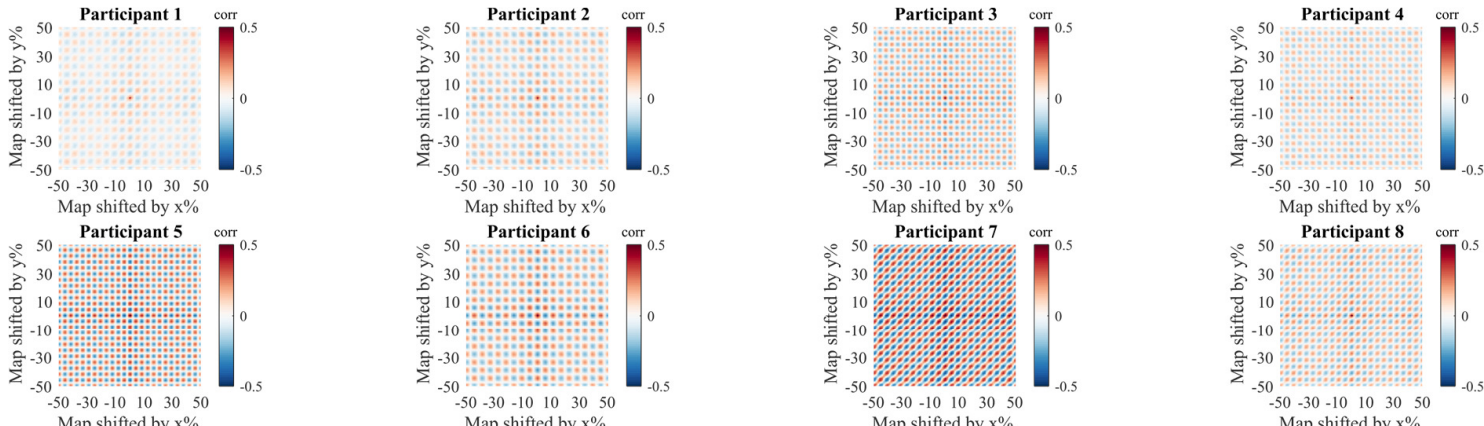
Map shifted by $x \%$

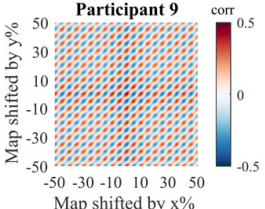

Map shifted by $\mathrm{x}$

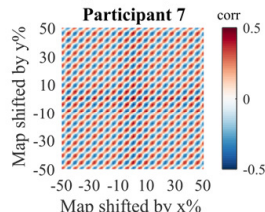

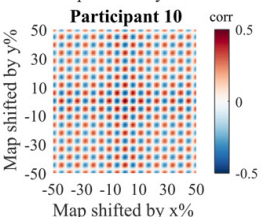

Map shifted by $x \%$

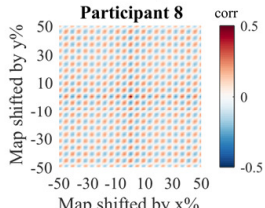




\section{Brain time periodicity spectra $\left(1^{\text {st }}\right.$ level $)$}
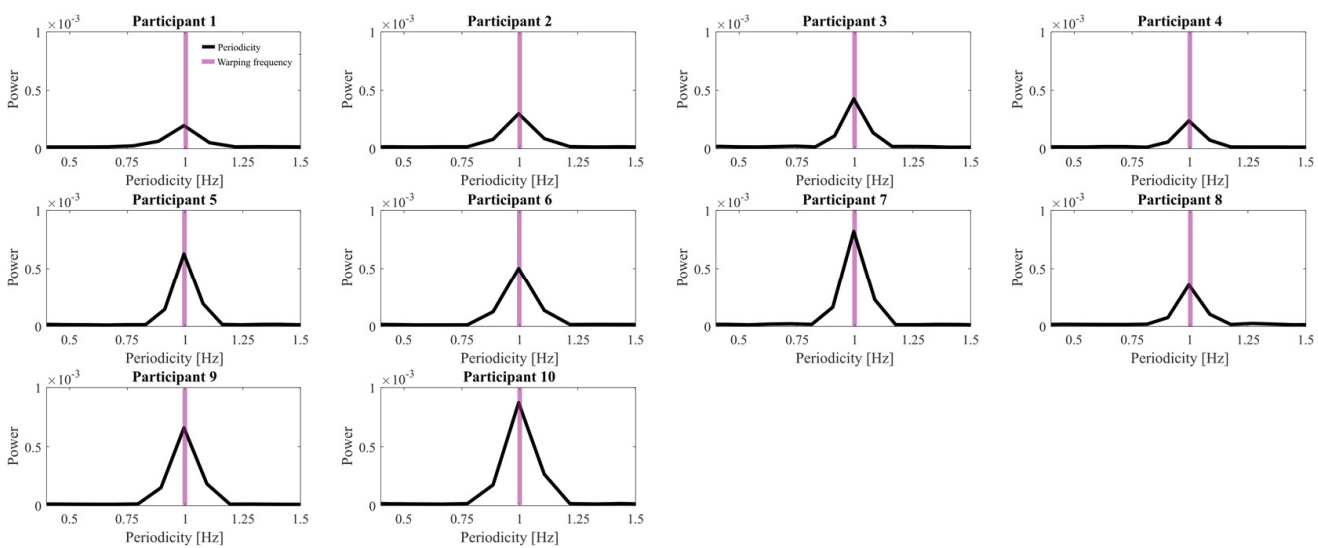

Brain time clusters

For the simulated dataset, we found few significant clusters with an alpha set at 0.05 and a critical t-value set at 1.96. We interpret these findings as indicating that the difference in simulated activity between left and right hemifield conditions is too small to be robustly detected by the classifier. However, since we know there is a difference in the ground truth between classes, we know this lack of significant results reflects a type II error. The fact that the periodicity analysis does show significant results indicates that, between empirical and permuted data, differences in the fluctuations of the neural signature are easier to detect than a mean baseline shift in signature fidelity.

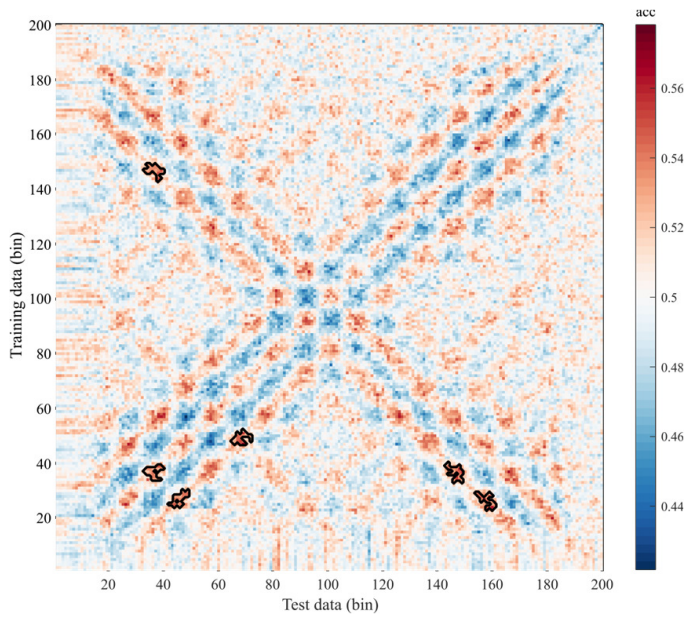




\section{Clock time TGM}
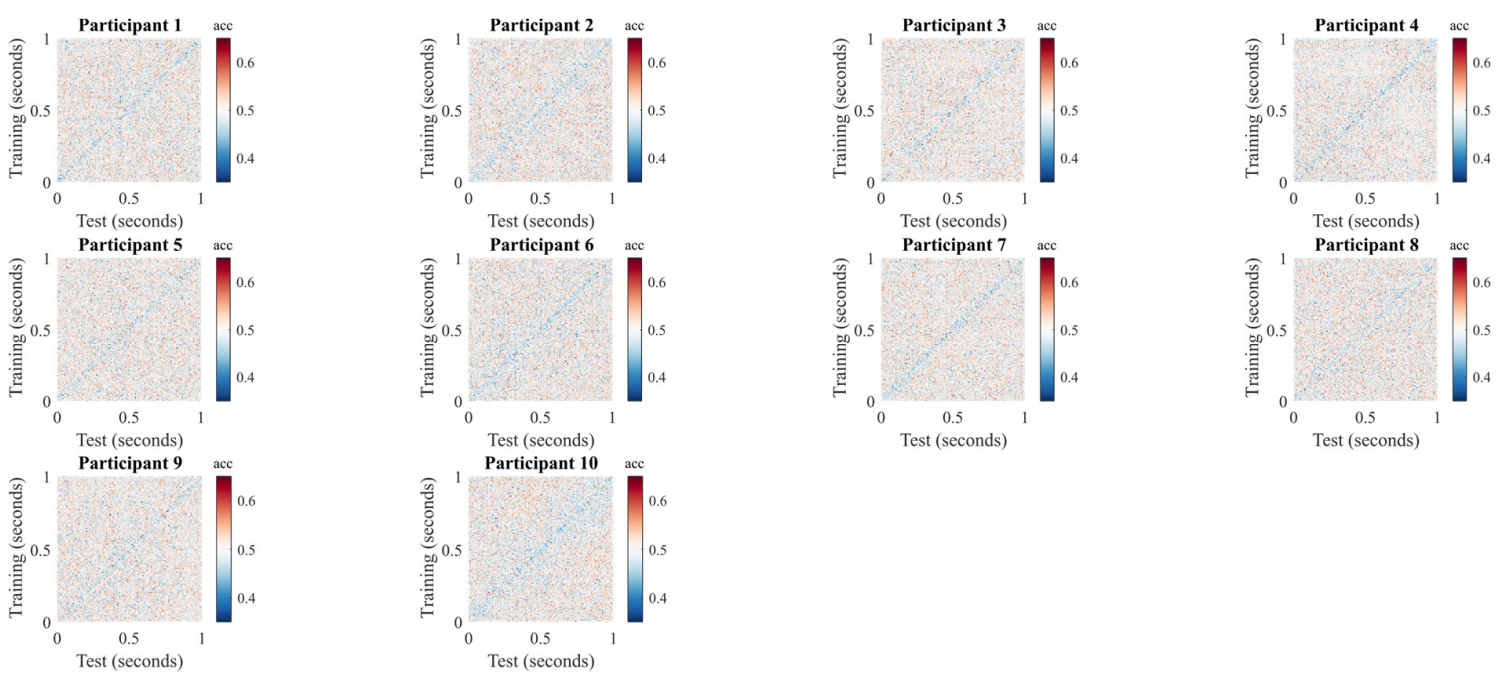

\section{Clock time autocorrelation}
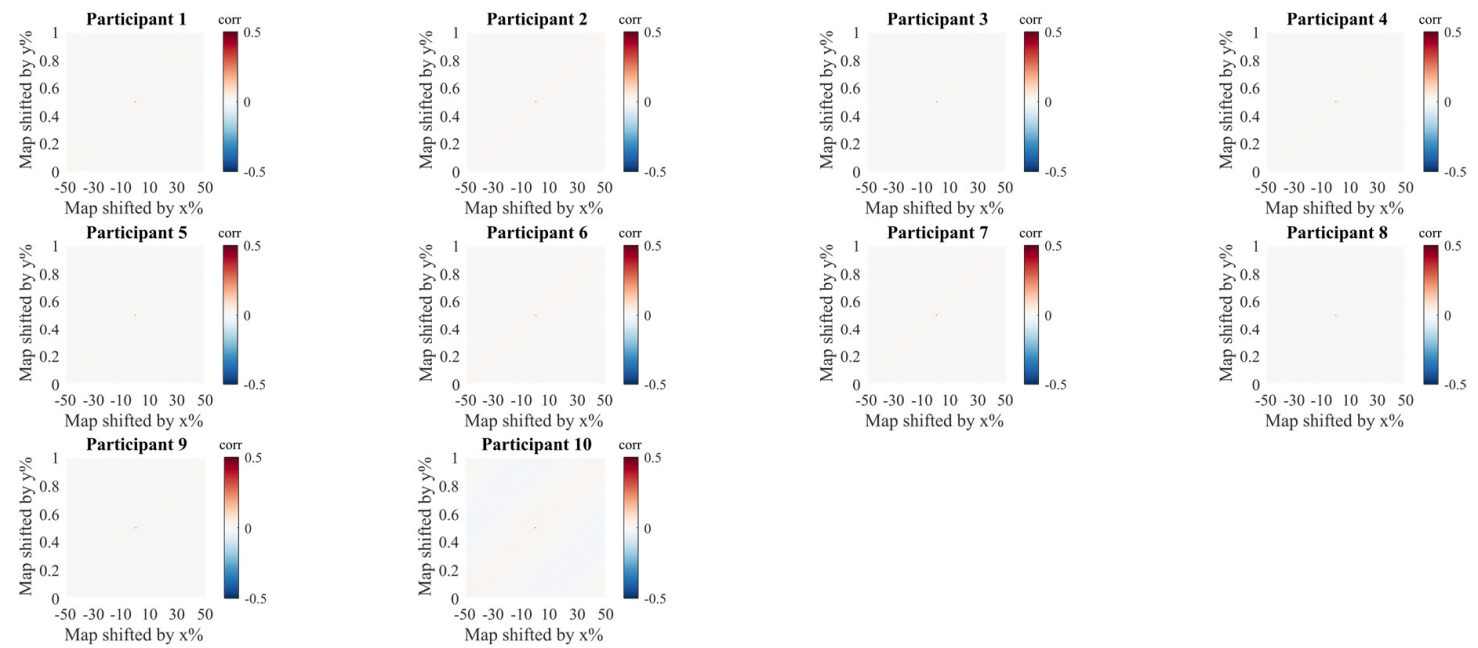

Clock time periodicity spectra $\left(1^{\text {st }}\right.$ level $)$
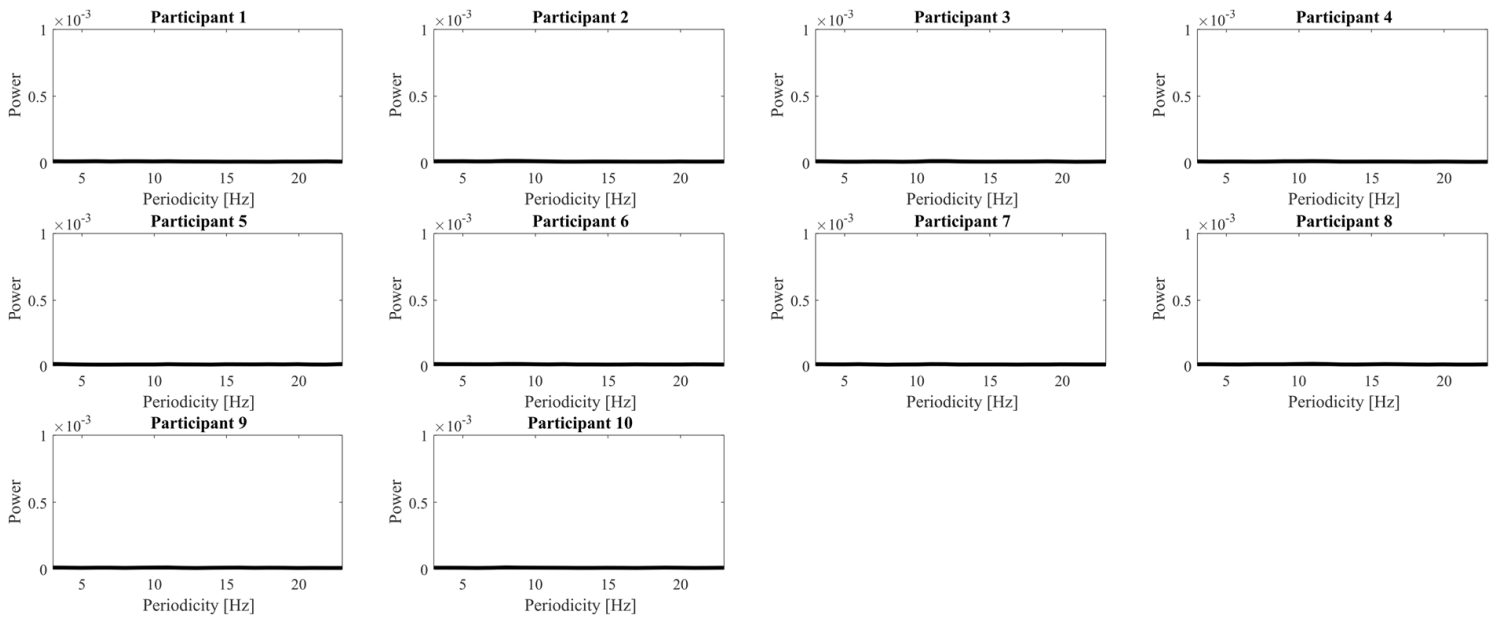


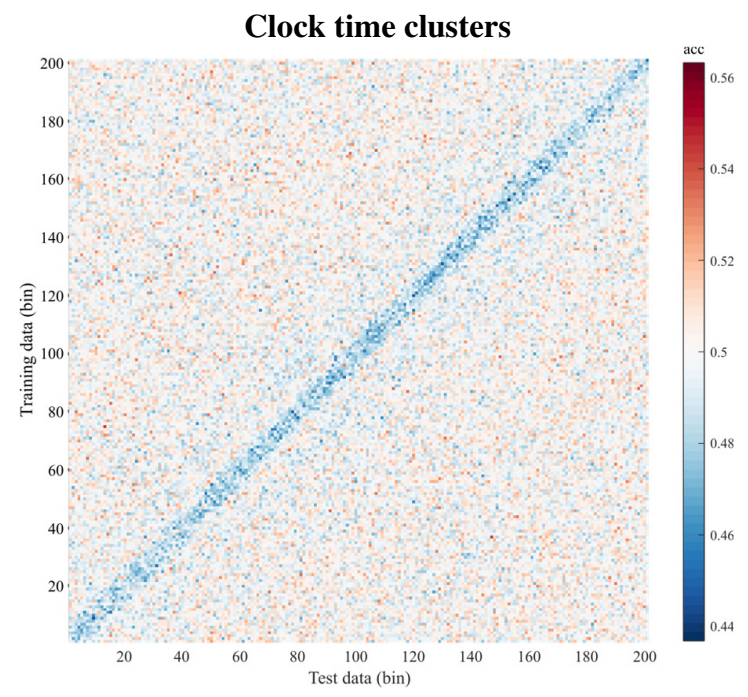

\subsubsection{Classification (control analysis)}

\section{Control brain time TGM}
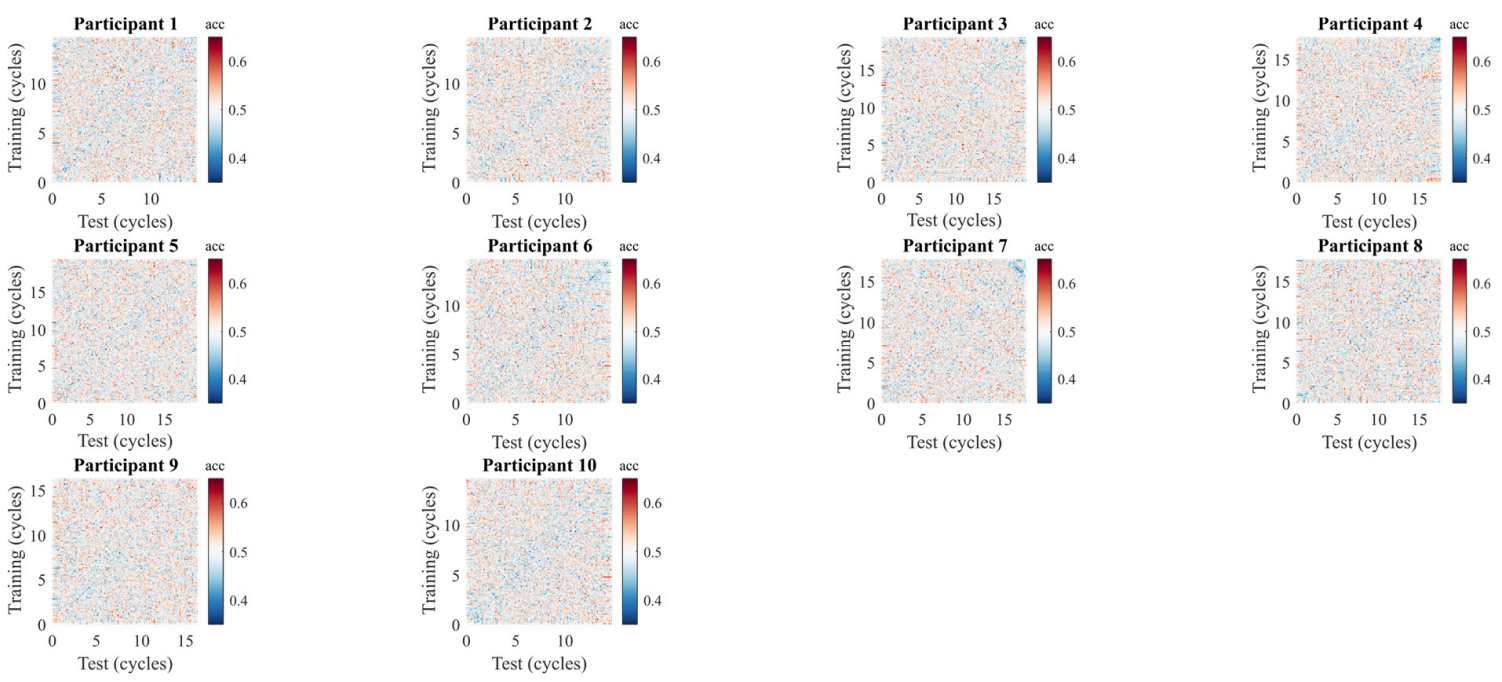

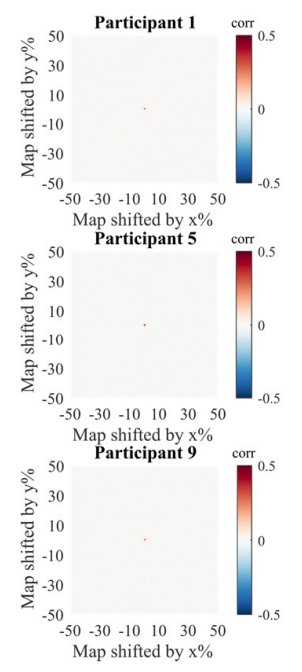

Control brain time autocorrelation
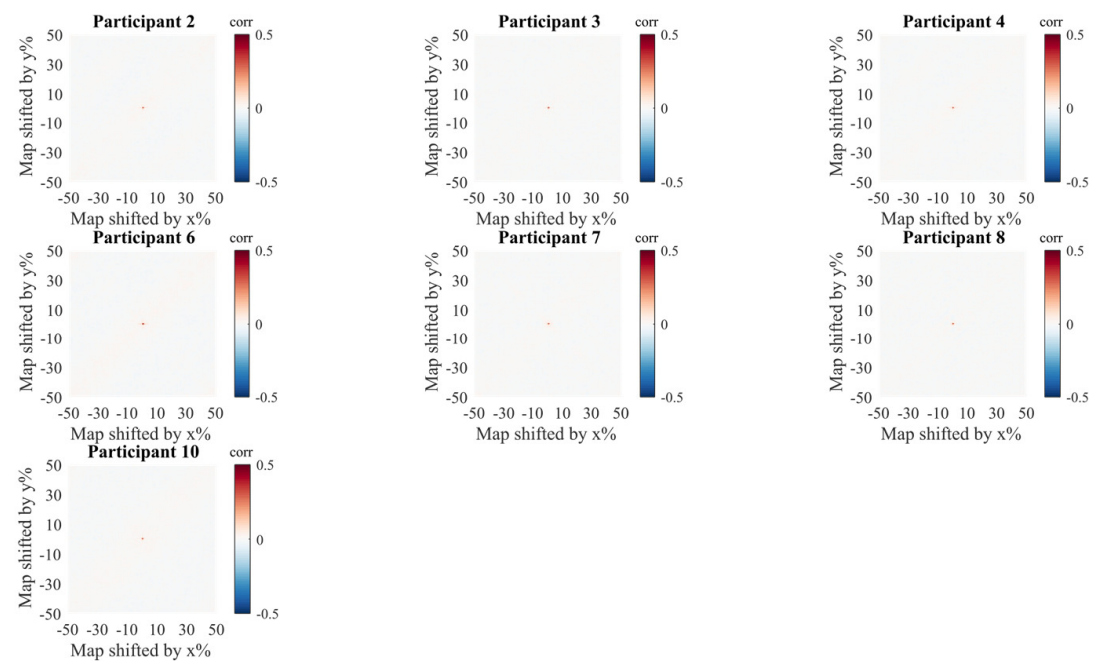

\section{Control brain time periodicity spectra ( $\left(1^{\text {st }}\right.$ level $)$}
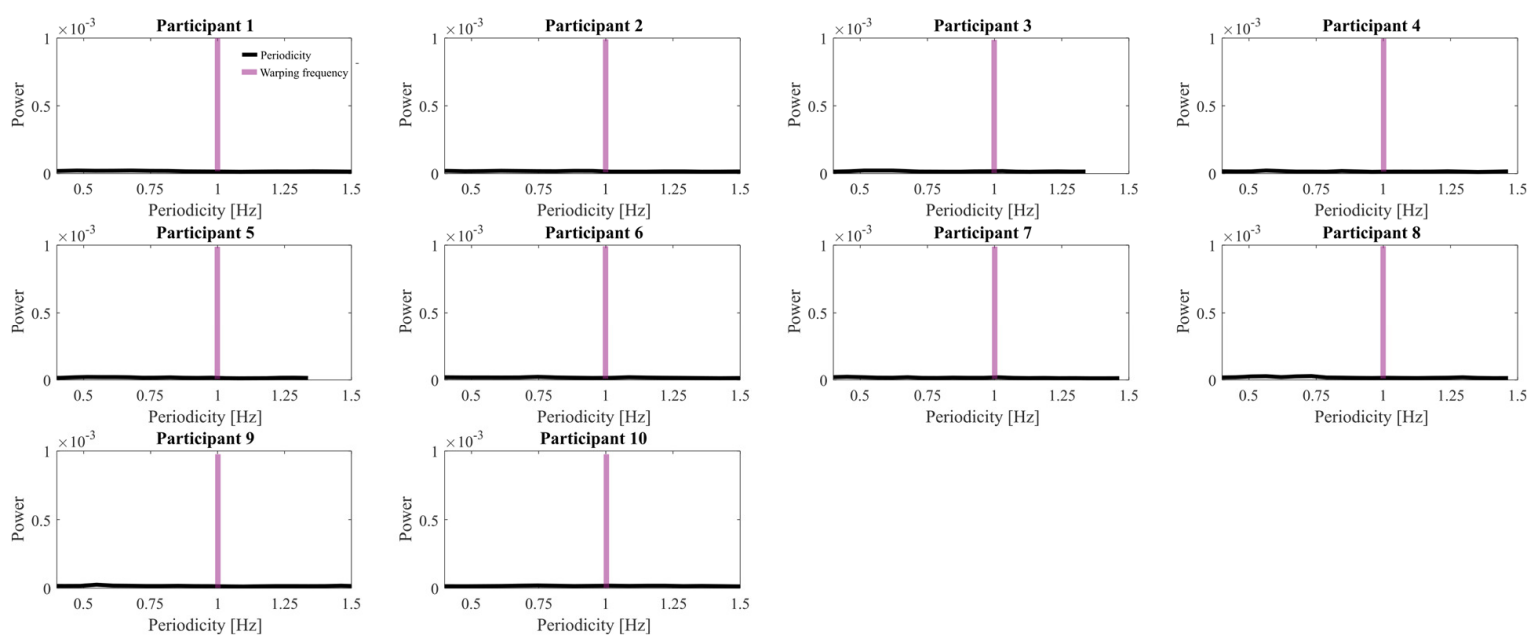

\section{Control brain time clusters}

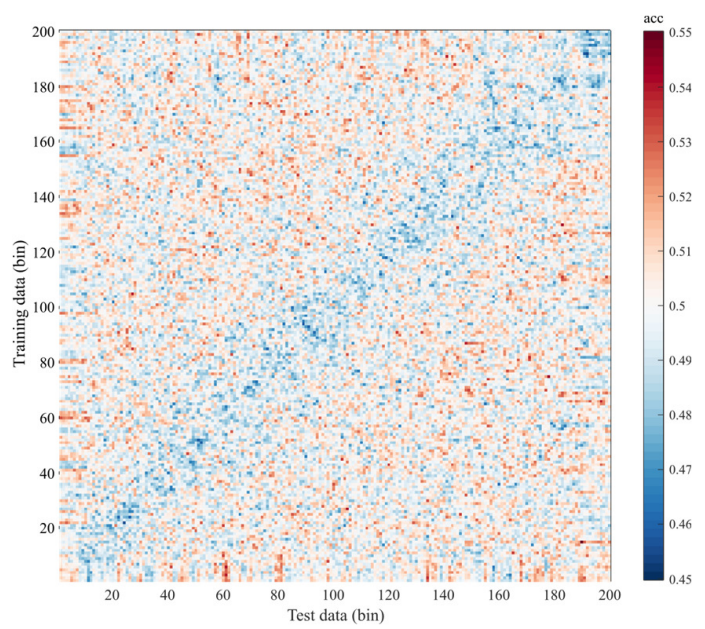




\subsection{Rodent}

3.2.1 Grid cells

Smoothed firing rate maps
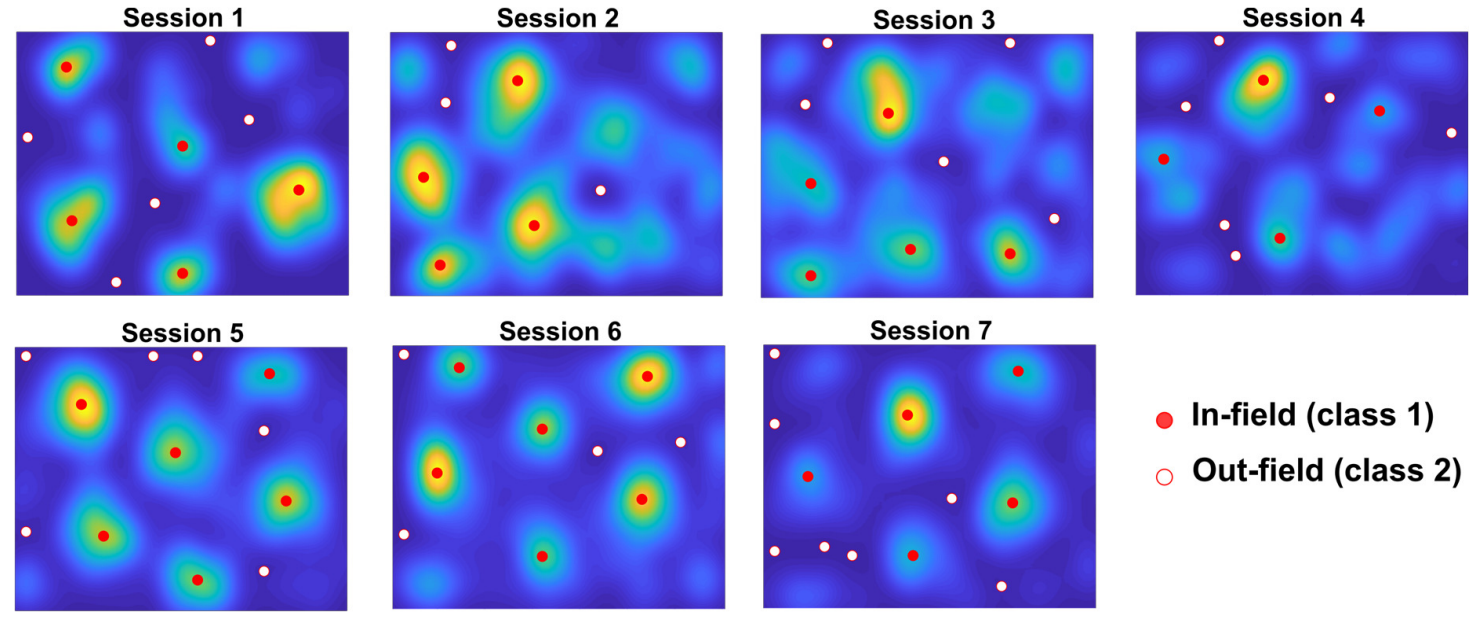

- In-field (class 1)

$\circ$ Out-field (class 2)

\subsubsection{Spike-field coupling}

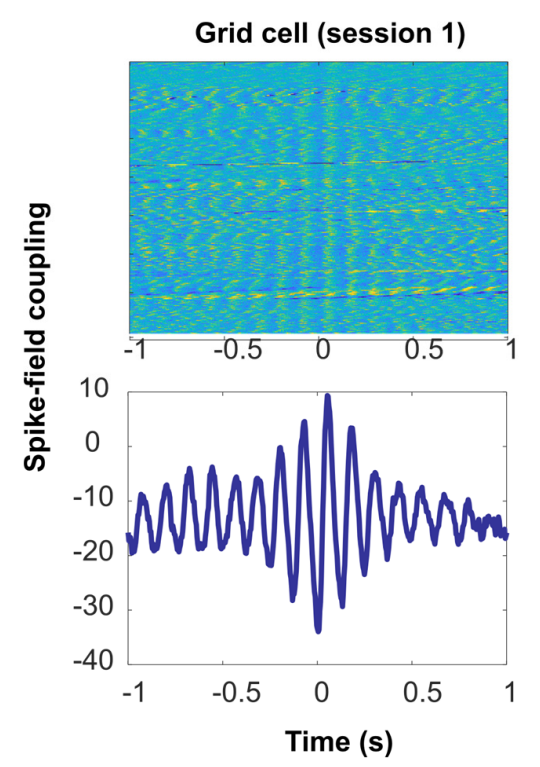

Grid cell (session 1)

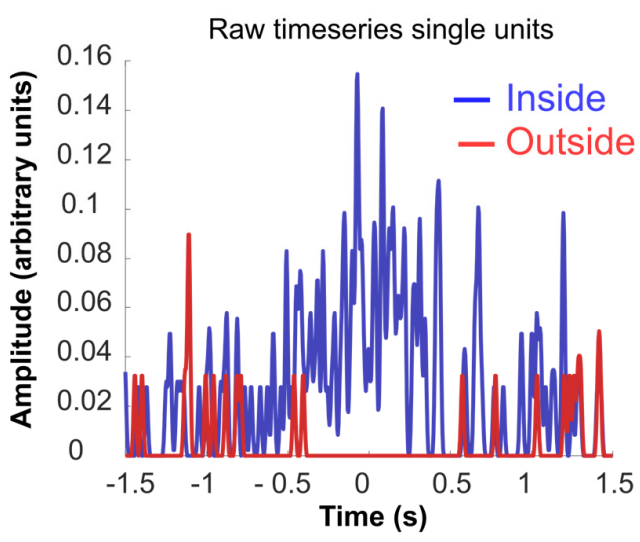




\subsubsection{Classification}

\section{Brain time TGM}
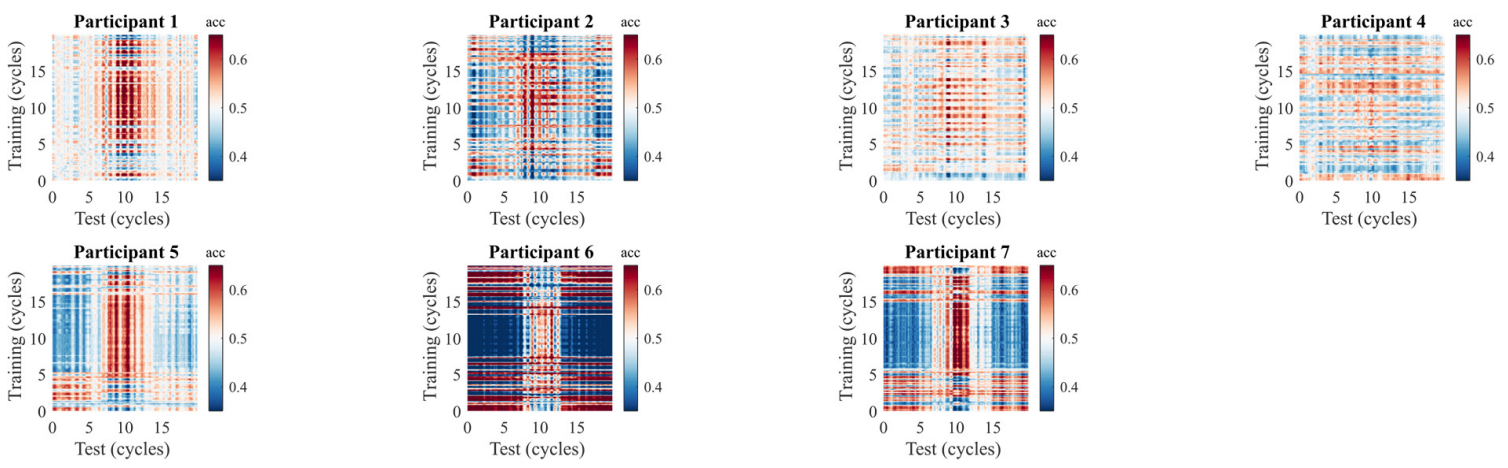

Brain time autocorrelation
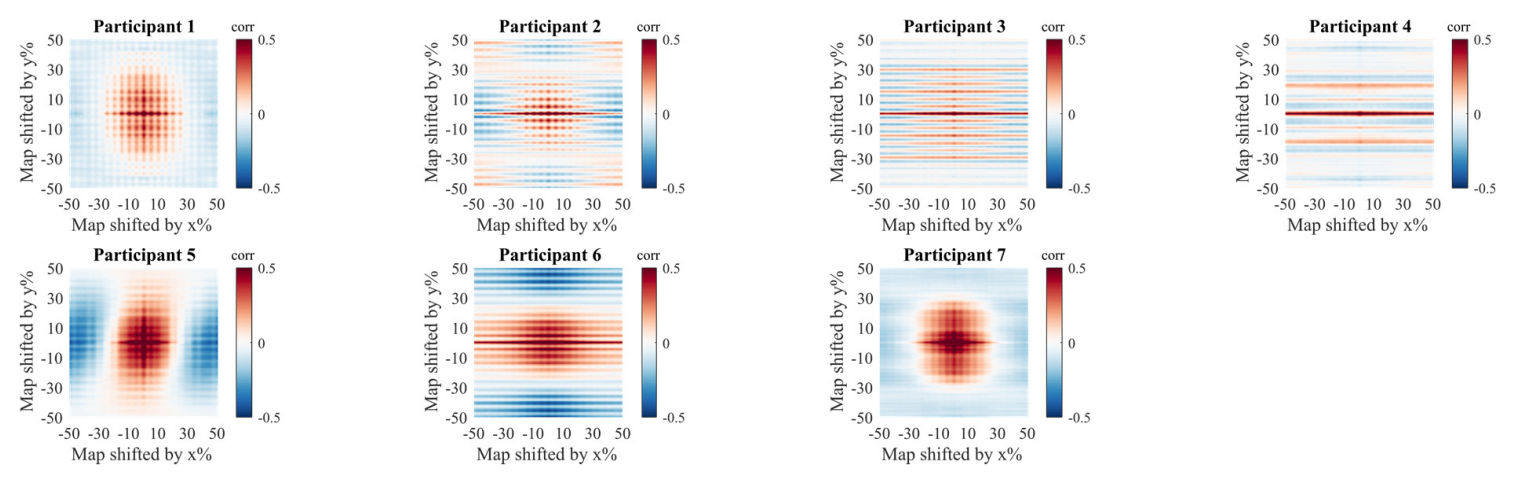

Brain time periodicity spectra $\left(1^{\text {st }}\right.$ level $)$
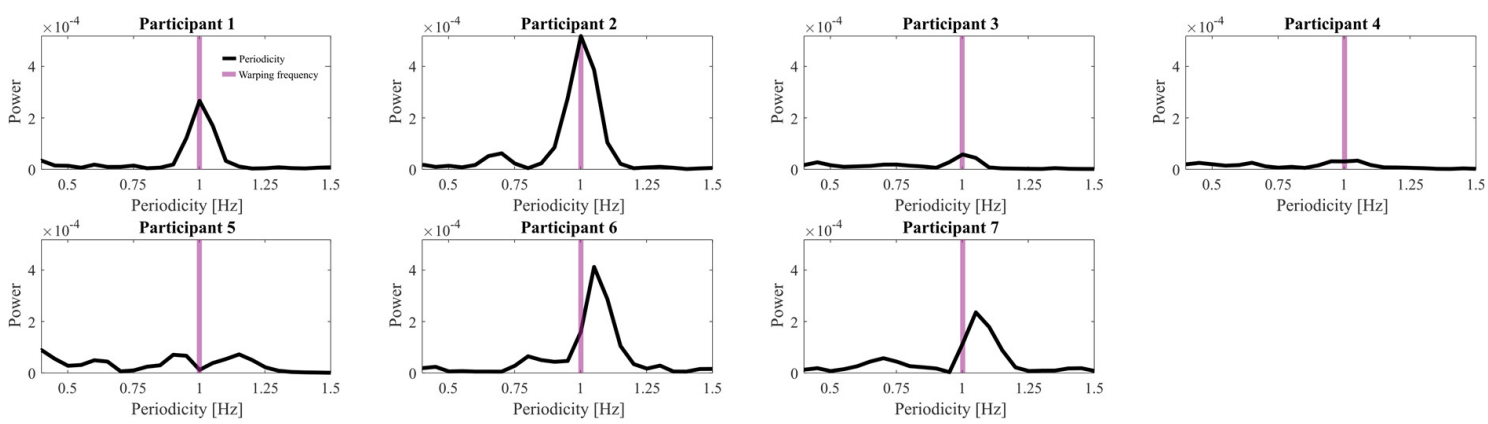


\section{Brain time clusters}

In line with our prediction, significant clusters occur in the period around $t=0$, where the data was defined to contain a class difference.

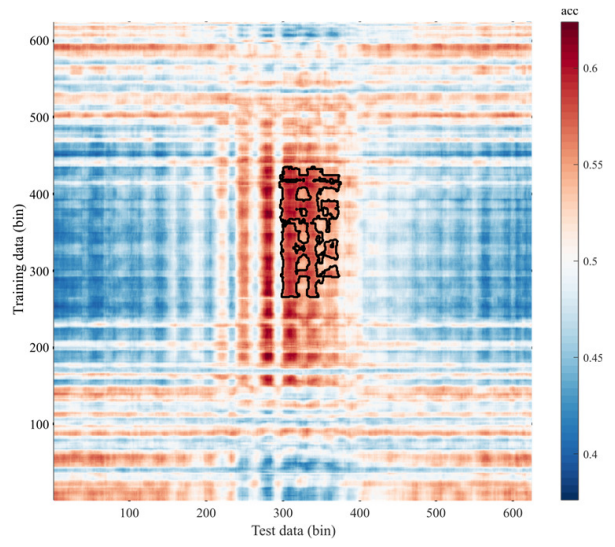

\section{Clock time TGM}
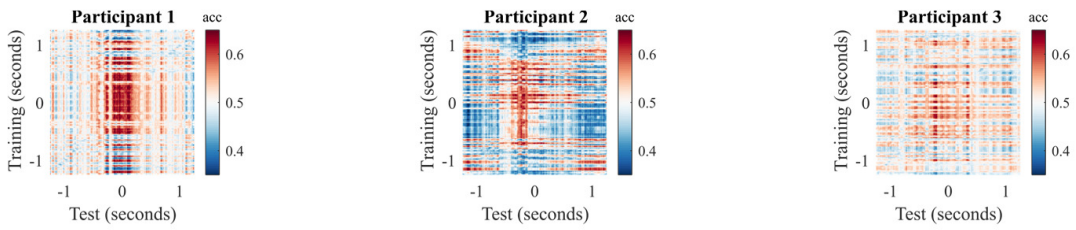

Participant 5
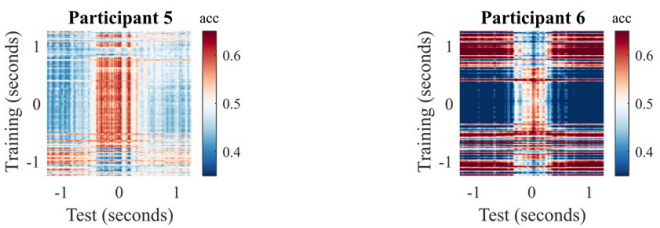

Participant 7
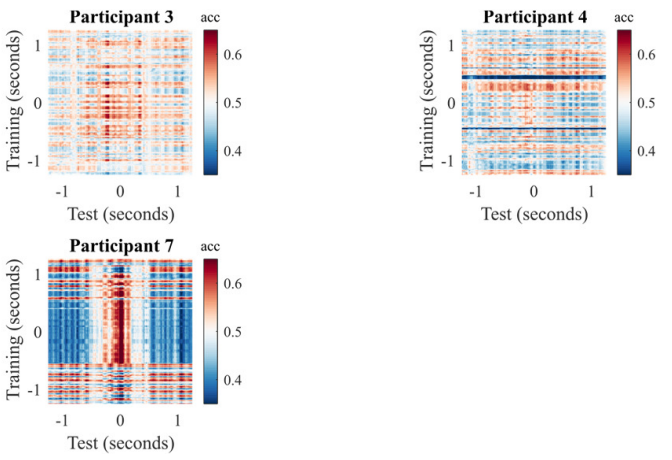

Clock time autocorrelation
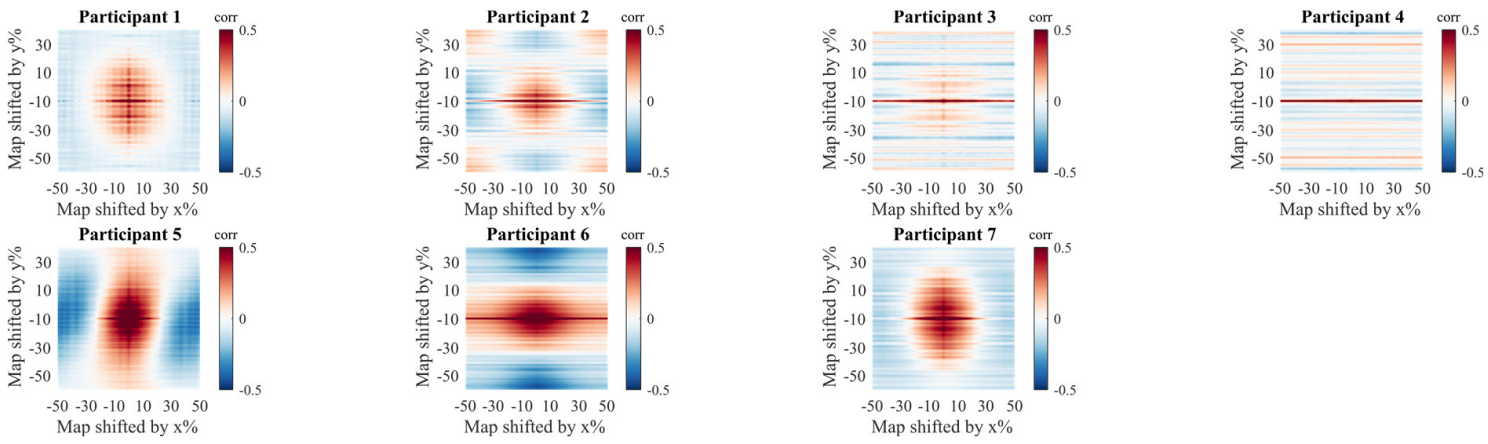

Map shifted by $\mathrm{x} \%$ 
Clock time periodicity spectra $\left(1^{\text {st }}\right.$ level $)$
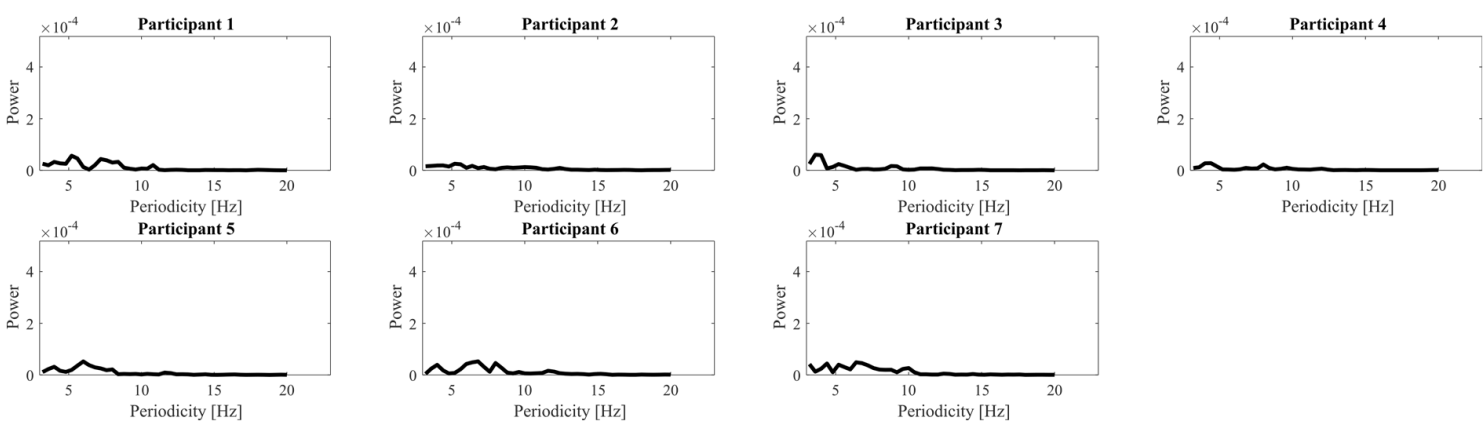

Clock time clusters

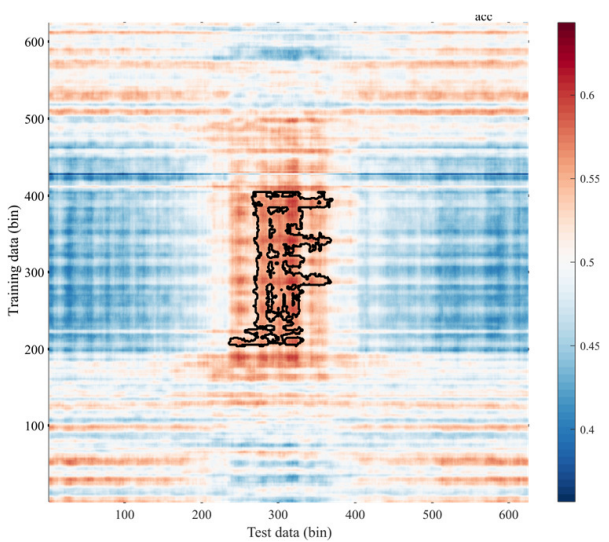




\subsection{Human}

\subsubsection{Classification}

\section{Brain time TGM}
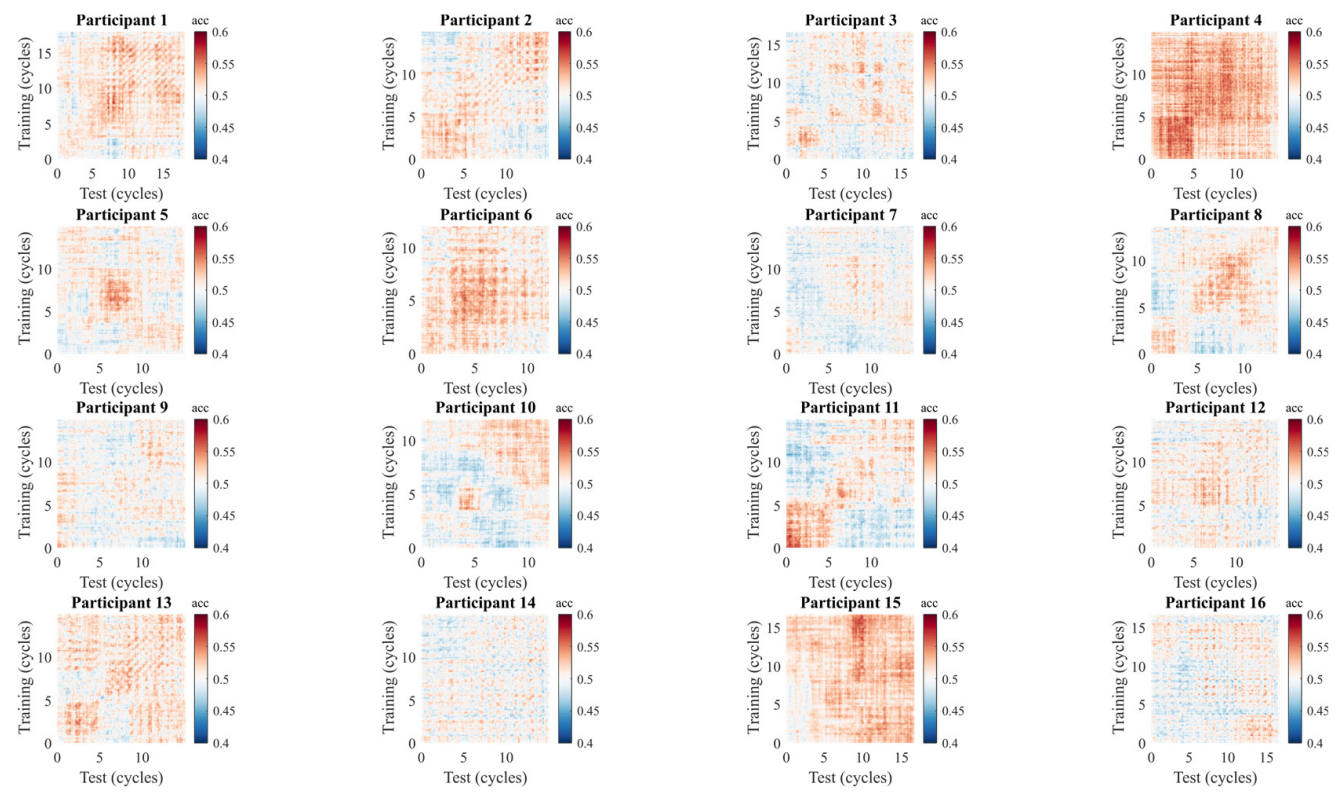

Brain time autocorrelation
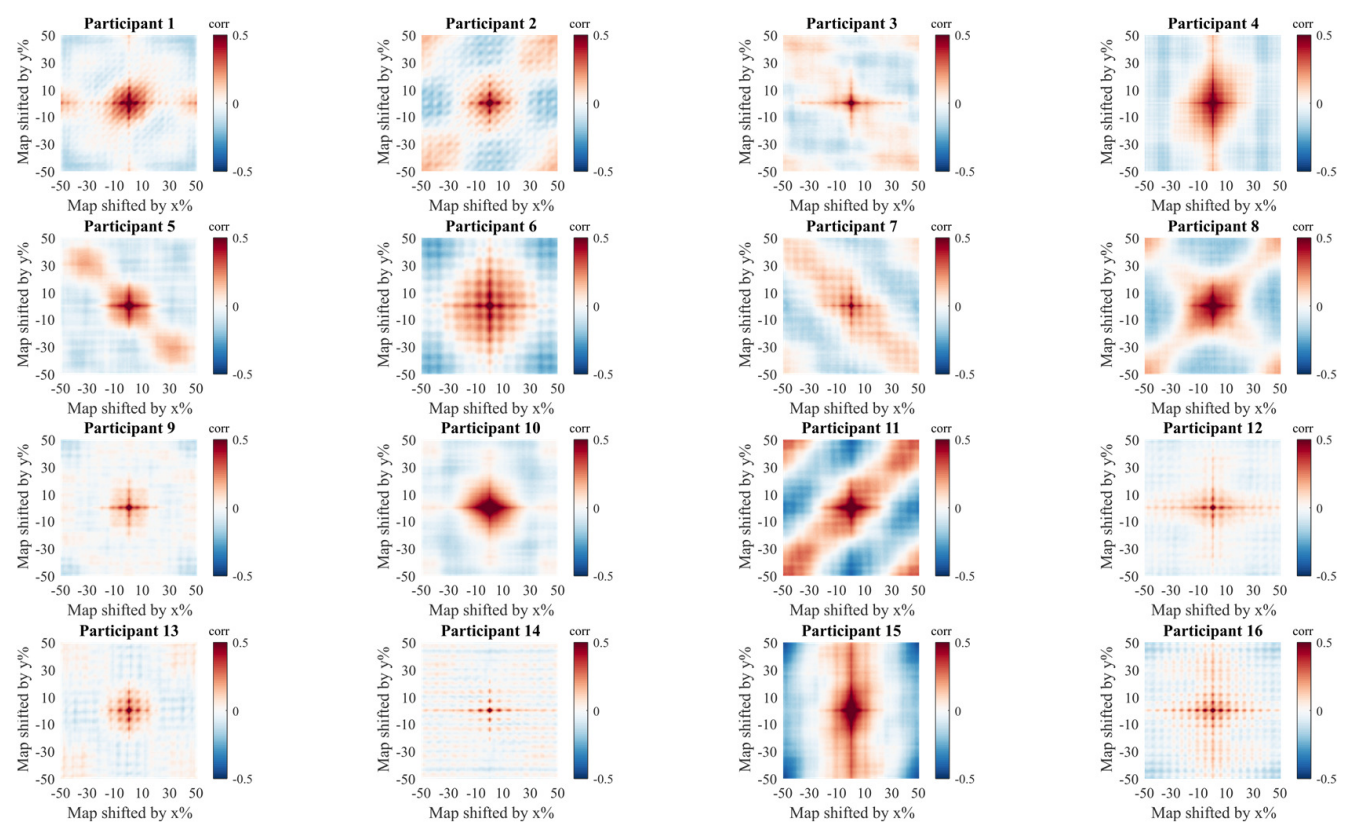
Brain time periodicity spectra $\left(1^{\text {st }}\right.$ level $)$
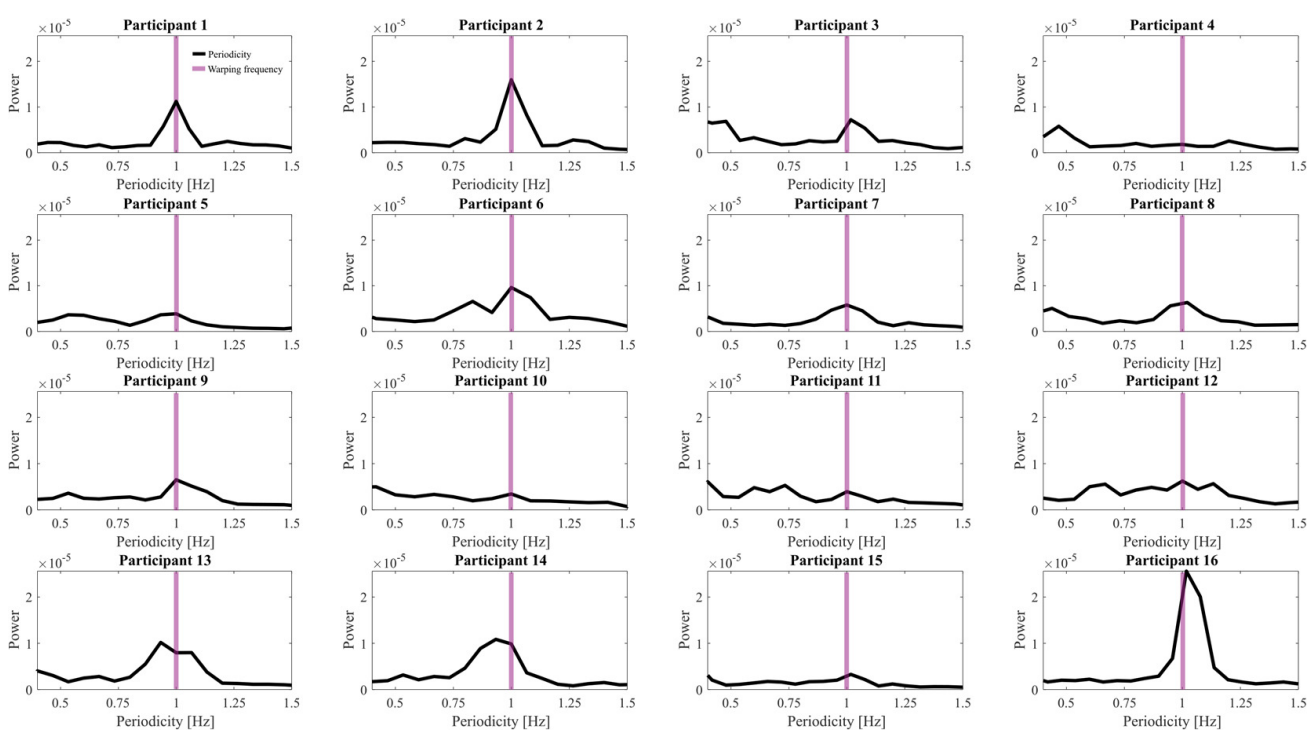

\section{Brain time clusters}

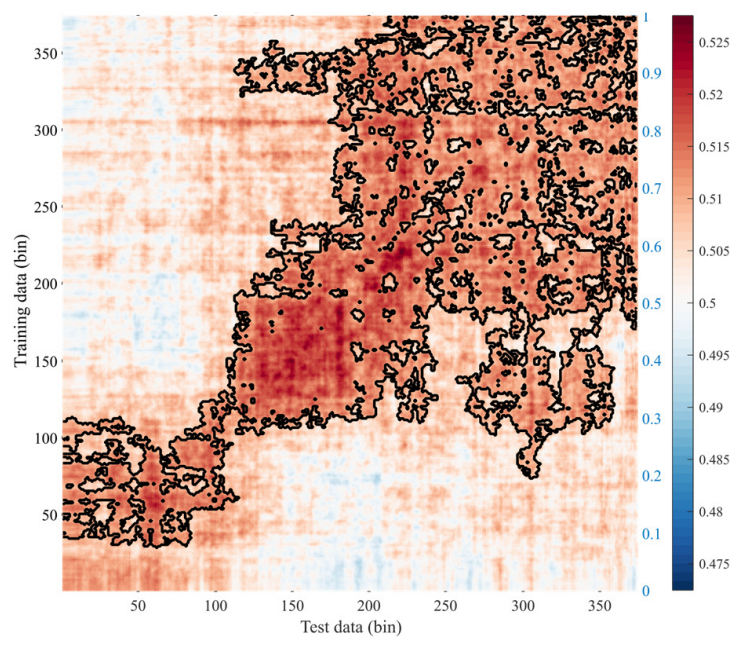


Clock time TGM
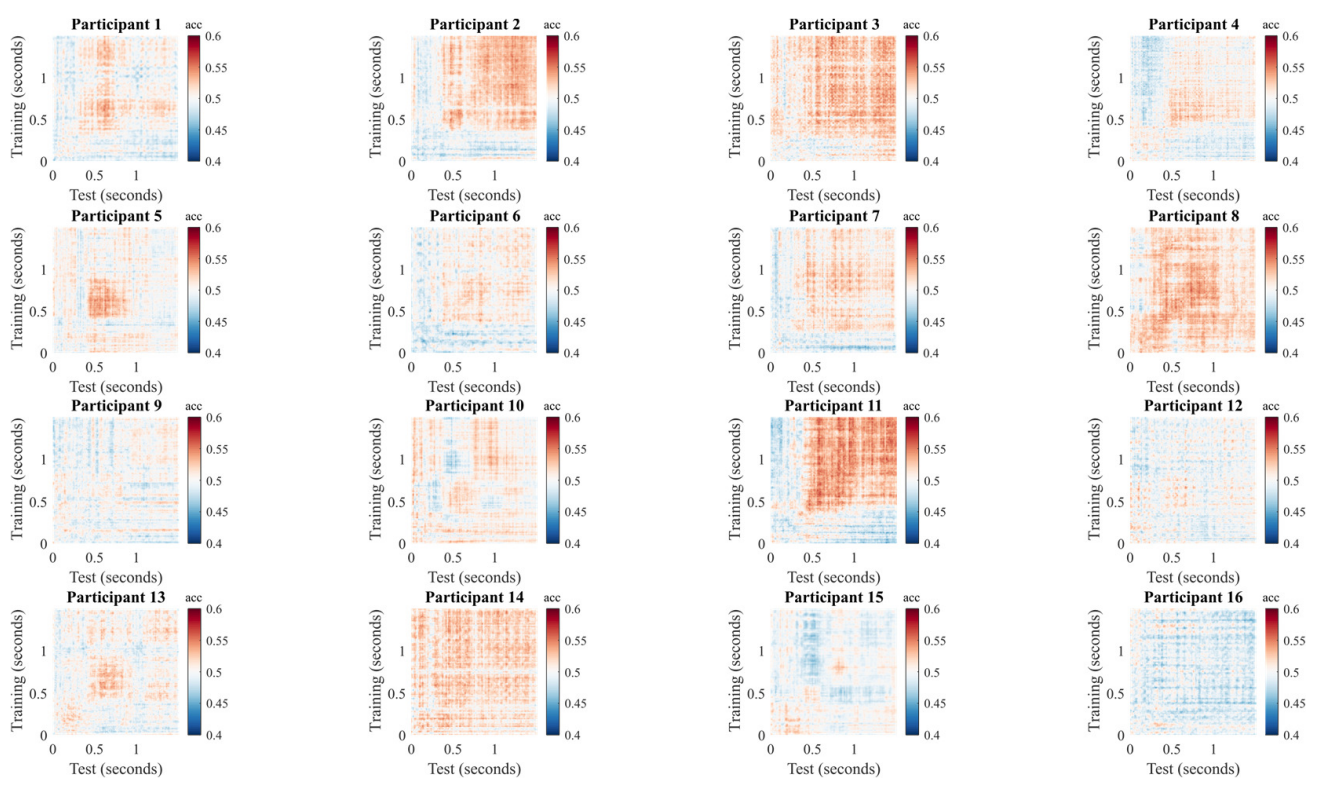

Clock time autocorrelation
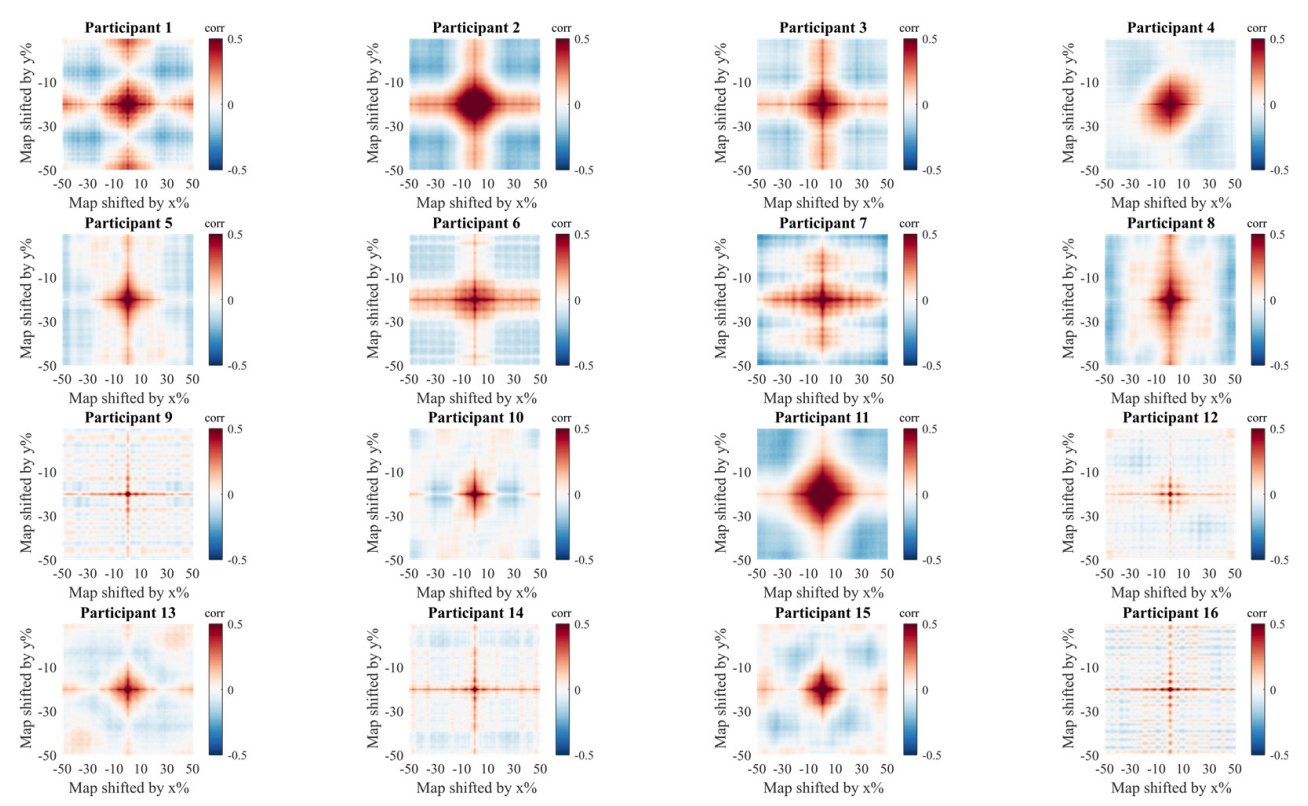
Clock time periodicity spectra $\left(1^{\text {st }}\right.$ level $)$
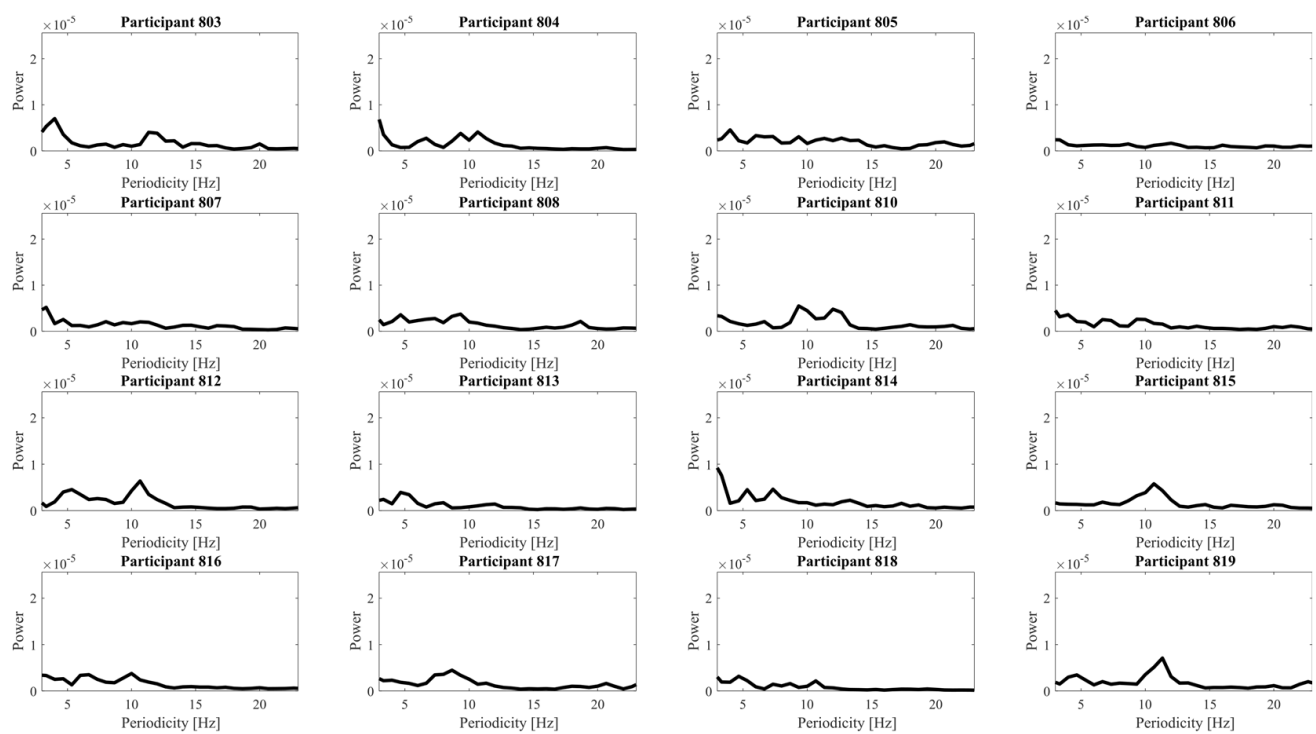

Clock time clusters

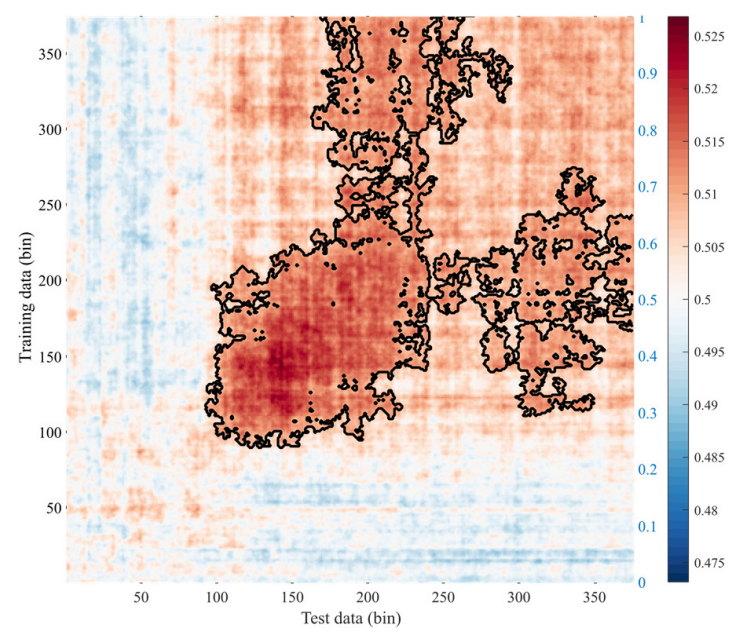




\section{Supplementary references}

Cohen, M. X. (2017). Multivariate cross-frequency coupling via generalized eigendecomposition. ELife, 6, e21792. https://doi.org/10.7554/eLife.21792

Belluscio, M. A., Mizuseki, K., Schmidt, R., Kempter, R., \& Buzsáki, G. (2012). Cross-Frequency Phase-Phase Coupling between Theta and Gamma Oscillations in the Hippocampus. The Journal of Neuroscience, 32(2), 423 LP - 435. https://doi.org/10.1523/JNEUROSCI.4122-11.2012

Benjamini, Y., \& Hochberg, Y. (1995). Controlling the False Discovery Rate: A Practical and Powerful Approach to Multiple Testing. Journal of the Royal Statistical Society: Series B (Methodological), 57(1), 289-300. https://doi.org/https://doi.org/10.1111/j.2517-6161.1995.tb02031.x

Berndt, D. J., \& Clifford, J. (1994). Using Dynamic Time Warping to Find Patterns in Time Series. Proceedings of the 3rd International Conference on Knowledge Discovery and Data Mining, 359-370.

Capotosto, P., Babiloni, C., Romani, G. L., \& Corbetta, M. (2009). Frontoparietal cortex controls spatial attention through modulation of anticipatory alpha rhythms. The Journal of Neuroscience: The Official Journal of the Society for Neuroscience, 29(18), 5863-5872. https://doi.org/10.1523/JNEUROSCI.053909.2009

van Driel, J., Olivers, C. N. L., \& Fahrenfort, J. J. (2021). High-pass filtering artifacts in multivariate classification of neural time series data. Journal of Neuroscience Methods, 352, 109080. https://doi.org/https://doi.org/10.1016/j.jneumeth.2021.109080

King, J.-R., \& Dehaene, S. (2014). Characterizing the dynamics of mental representations: the temporal generalization method. Trends in Cognitive Sciences, 18(4), 203-210. https://doi.org/https://doi.org/10.1016/j.tics.2014.01.002

Makeig, S., Bell, A. J., Jung, T.-P., \& Sejnowski, T. J. (1995). Independent Component Analysis of Electroencephalographic Data. Proceedings of the 8th International Conference on Neural Information Processing Systems, 145-151.

Maris, E., \& Oostenveld, R. (2007). Nonparametric statistical testing of EEG- and MEG-data. Journal of Neuroscience Methods, 164(1), 177-190. https://doi.org/10.1016/j.jneumeth.2007.03.024

Newman, E. L., \& Hasselmo, M. E. (2014). Grid cell firing properties vary as a function of theta phase locking preferences in the rat medial entorhinal cortex. In Frontiers in Systems Neuroscience (Vol. 8, p. 193). https://www.frontiersin.org/article/10.3389/fnsys.2014.00193

Oostenveld, R., Fries, P., Maris, E., \& Schoffelen, J.-M. (2011). FieldTrip: Open Source Software for Advanced Analysis of MEG, EEG, and Invasive Electrophysiological Data. Computational Intelligence and Neuroscience, 2011, 156869. https://doi.org/10.1155/2011/156869

Parra, L. C., Spence, C. D., Gerson, A. D., \& Sajda, P. (2005). Recipes for the linear analysis of EEG. NeuroImage, 28(2), 326-341. https://doi.org/10.1016/j.neuroimage.2005.05.032

Pletzer, B., Kerschbaum, H., \& Klimesch, W. (2010). When frequencies never synchronize: the golden mean and the resting EEG. Brain Research, 1335, 91-102. https://doi.org/10.1016/j.brainres.2010.03.074

Sakoe, H., \& Chiba, S. (1978). Dynamic programming algorithm optimization for spoken word recognition. IEEE Transactions on Acoustics, Speech, and Signal Processing, 26(1), 43-49. https://doi.org/10.1109/TASSP.1978.1163055

Treder, M. S. (2020). MVPA-Light: A Classification and Regression Toolbox for Multi-Dimensional Data. In Frontiers in Neuroscience (Vol. 14, p. 289). https://www.frontiersin.org/article/10.3389/fnins.2020.00289 